\title{
Interval Generalized Ordered Weighted Utility Multiple Averaging Operators and Their Applications to Group Decision-Making
}

\author{
Yunna $\mathrm{Wu}{ }^{1,2}$, Chuanbo Xu ${ }^{1,2, *}$, Haobo Zhang ${ }^{1}$ and Jianwei Gao ${ }^{1}$ \\ 1 School of Economics and Management, North China Electric Power University, Beijing 102206, China; \\ ncepuwuyunna@126.com (Y.W.); 1101340119@ncepu.edu.cn (H.Z.); 50601210@ncepu.edu.cn (J.G.) \\ 2 Beijing Key Laboratory of New Energy and Low-Carbon Development, North China Electric \\ Power University, Changping, Beijing 102206, China \\ * Correspondence: 18810736266@163.com; Tel.: +86-188-1073-6266
}

Academic Editor: Angel Garrido

Received: 22 March 2017; Accepted: 28 June 2017; Published: 1 July 2017

\begin{abstract}
We propose a new class of aggregation operator based on utility function and apply them to group decision-making problem. First of all, based on an optimal deviation model, a new operator called the interval generalized ordered weighted utility multiple averaging (IGOWUMA) operator is proposed, it incorporates the risk attitude of decision-makers (DMs) in the aggregation process. Some desirable properties of the IGOWUMA operator are studied afterward. Subsequently, under the hyperbolic absolute risk aversion (HARA) utility function, another new operator named as interval generalized ordered weighted hyperbolic absolute risk aversion utility multiple averaging-HARA (IGOWUMA-HARA) operator is also defined. Then, we discuss its families and find that it includes a wide range of aggregation operators. To determine the weights of the IGOWUMA-HARA operator, a preemptive nonlinear objective programming model is constructed, which can determine a uniform weighting vector to guarantee the uniform standard comparison between the alternatives and measure their fair competition under the condition of valid comparison between various alternatives. Moreover, a new approach for group decision-making is developed based on the IGOWUMA-HARA operator. Finally, a comparison analysis is carried out to illustrate the superiority of the proposed method and the result implies that our operator is superior to the existing operator.
\end{abstract}

Keywords: group decision-making; aggregation operator; weight; utility function

\section{Introduction}

Multiple attributes decision-making (MADM) refers to considering the problem of evaluating or selecting alternatives that are associated with incommensurate and conflicting criteria. However, the increasing complexity of the socioeconomic environment makes it less possible for a single decision-maker (DM) to consider all relevant aspects of a problem as many decision-making processes take place in group settings. As a result, multiple attributes group decision-making (MAGDM) has been receiving more and more attentions from researchers and has been extensively applied to our daily life [1-7]. Nevertheless, due to the complexities of objects and the vagueness of human mind, it is more appropriate for DMs to use interval/uncertain information than other descriptors to express their assessments in the actual process of MAGDM.

The objective of interval MAGDM (IMAGDM) is to find the optimal solution(s) from a set of feasible alternatives by means of interval information provided by DMs. To realize this objective, the construction of aggregating operators and the determination of aggregation operators' weights are the two key issues. 
A variety of operators have been developed in the past few decades, among them, ordered weighted averaging (OWA) operators introduced by Yager [8] are the most widely used ones. Motivated by these, an extension of the OWA operator is the uncertain OWA (UOWA) operator [9], which aggregates the input arguments taking the form of intervals rather than exact numbers. Some new developments about it have mostly appeared from 2008 to 2013. For example, Xu [10] proposed dependent uncertain ordered weighted averaging (DUOWA) operators and dependent uncertain ordered weighted geometric (DUOWG) operators. Xu and Yager [11] introduced the uncertain power ordered weighted geometric (UPOWG) operator on the basis of the power average operator and the geometric mean. Merigó and Casanovas [12] proposed uncertain induced quasi-arithmetic OWA (Quasi-UIOWA) operator which uses the main characteristics of the induced OWA, the quasi-arithmetic OWA and the UOWA operator. Merigo and Casanovas [13] presented the uncertain heavy OWA (UHOWA) operator and uncertain induced heavy OWA (UIHOWA) operator. $\mathrm{Xu}$ and Cai [14] developed the uncertain power ordered weighted average (UPOWA) operator on the basis of the power averaging (PA) operator and the UOWA operator. To extend the generalized ordered weighted averaging operator (GOWA), Zhou et al. [15] developed the uncertain generalized ordered weighted averaging (UGOWA) operator. Zeng et al. [16] present the uncertain probabilistic ordered weighted averaging distance (UPOWAD) operator which uses distance measures in a unified framework between the probability and the OWA operator that considers the degree of importance of each concept in the aggregation. However, the above uncertain aggregation operators only focus on using the mean to eliminate the difference of variable values but fail to consider DMs' risk attitude in the aggregation process. In actual decision making, different DMs may show different risk attitudes, and the risk attitudes of the same DM may also vary at different times. Obviously, ignoring the risk attitude of DMs will lead to a wrong decision-making.

Another important issue of applying the aggregation operator for IMAGDM is how to determine the associated weights. Many researchers have focused on this issue and developed some useful approaches for determining weights [9,10,15,17-19]. For instance, $\mathrm{Xu}$ and Da [9] established a linear objective-programming model to generate the UOWA weights by utilizing the given partial weight information, the input interval arguments, and the associated aggregated values. Ahn [18] presented several weighting functions having constant values of orness irrespective of the number of objectives aggregated. Considering the impact of optimism degree of DMs on the decision-making results and by applying the absolute deviation measure, $\mathrm{Xu}$ [10] derived the weights of DUOWA operator which only depend on the aggregated interval arguments. To determine UGOWA weights, Zhou et al. [15] used the relative deviation measure to construct a nonlinear model. However, we find that they did not provide a uniform weighting vector based on the weighting models in $[10,15]$, which can affect the accuracy of the decision results and lead to unreasonable results.

Therefore, the aim of this paper is to develop some interval utility aggregation operators to deal with interval preference information, which incorporates the risk attitude of DMs in the aggregation process. Under the general framework of utility function, a new operator called the interval generalized ordered weighted utility multiple averaging (IGOWUMA) operator by applying the penalty theory is derived firstly, and then by studying its properties we find that it is commutative, idempotent, monotone increasing and bounded. Furthermore, we focus on a concrete utility function-hyperbolic absolute risk aversion (HARA), which is rather rich, e.g., by suitable adjustments of the parameters one can respectively obtain power utility, exponential utility and logarithm utility. Under the HARA utility, we propose another new operator named as the interval generalized ordered weighted hyperbolic absolute risk aversion utility multiple averaging-HARA (IGOWUMA-HARA) operator, and investigate its families which include a wide range of aggregation operators such as interval generalized ordered weighted multiple averaging (IGOWMA) operator, interval ordered weighted multiple averaging (IOWMA) operator and interval ordered weighted geometric averaging (IOWGA) operator. The merits of the IGOWUMA-HARA operator are that it not only reflects the DMs' risk attitude towards the aggregation information, but also provides a very general formula including a wide range 
of aggregation operators. To determine the IGOWUMA-HARA operator' weights, we propose a preemptive nonlinear objective programming model, which can provide a uniform weighting vector to guarantee the uniform standard comparison between the alternatives and reduce the influence of unfair arguments on the aggregated results. After that, based on the IGOWUMA-HARA operator and weight determination model, a new approach for IMAGDM is developed. This approach is also effectively applicable to different group decision-making problems such as engineering management and financial management, etc. Finally, an example of the investment selection is provided to examine our approach.

The rest of the paper is organized as follows. Section 2 reviews some basic concepts. Section 3 presents the IGOWUMA operator and analyzes its properties as well as provides an IGOWUMA-HARA operator and identifies its families. We then present a preemptive nonlinear objective programming model for determining IGOWUMA-HARA weights in Section 4. Section 5 develops an approach for IMAGDM under the IGOWUMA-HARA operator. An illustrative example is provided in Section 6 and the conclusions are drawn in Section 7.

\section{Preliminaries}

This section briefly reviews definitions and operations of interval values, UGOWA operator and HARA utility function.

\subsection{Definitions and Operations of Interval Values}

Moore [20] presented the interval number. It can be defined as follows.

Definition 1. Let $\widetilde{x}=\left[x^{L}, x^{U}\right]=\left\{x \mid 0 \leq x^{L} \leq x^{U}\right\}$, then $\widetilde{x}$ is called a nonnegative interval number. Especially, $\widetilde{x}$ is a nonnegative real number as $\widetilde{x}^{L}=\widetilde{x}^{U}$.

To simplify the calculation, throughout this paper, let $\Omega=\left\{\widetilde{x} \mid \widetilde{x}=\left[x^{L}, x^{U}\right], 0 \leq x^{L} \leq x^{U}\right\}$ be the set of all nonnegative interval numbers.

Definition 2. [10,11] Let $\widetilde{x}, \widetilde{z} \in \Omega, \widetilde{x}=\left[x^{L}, x^{U}\right], \widetilde{z}=\left[z^{L}, z^{U}\right]$, then

(1) $\widetilde{x}=\widetilde{z}$, if $x^{L}=z^{L}$ and $x^{U}=z^{U}$.

(2) $\tilde{x}+\widetilde{z}=\left[x^{L}+z^{L}, x^{U}+z^{U}\right]$.

(3) $\tilde{x} \cdot \widetilde{z}=\left[x^{L} \cdot z^{L}, x^{U} \cdot z^{U}\right]$.

(4) $\tilde{x} / \widetilde{z}=\left[x^{L} / z^{U}, x^{U} / z^{L}\right]$.

(5) $k \widetilde{x}=\left[k x^{L}, k x^{U}\right]$.

(6) If $k \geq 0$, then $\widetilde{x}^{k}=\left[\left(x^{L}\right)^{k},\left(x^{U}\right)^{k}\right]$, and if $k \leq 0$, then $\widetilde{x}^{k}=\left[\left(x^{U}\right)^{k},\left(x^{L}\right)^{k}\right]$.

(7) If $k \geq 1$, then $\log _{k} \tilde{x}=\left[\log _{k} x^{L}, \log _{k} x^{U}\right]$, and if $0<k<1$, then $\log _{k} \widetilde{x}=\left[\log _{k} x^{U}, \log _{k} x^{L}\right]$.

$\mathrm{Xu}$ and $\mathrm{Da}[8]$ presented a possibility degree formula for the comparison between interval numbers, which is shown as follows:

Definition 3. Let $\widetilde{x}=\left[x^{L}, x^{U}\right] \in \Omega$ and $\widetilde{z}=\left[z^{L}, z^{U}\right] \in \Omega$ be two interval numbers, and $l_{\widetilde{x}}=x^{U}-x^{L}$, $l_{\widetilde{z}}=z^{U}-z^{L}$, then the degree of possibility of $\widetilde{x} \geq \widetilde{z}$ is defined as

$$
p(\widetilde{x} \geq \widetilde{z})=\max \left\{1-\max \left(\left[\widetilde{z}^{U}-\widetilde{x}^{L}\right] /\left[l_{\widetilde{x}}+l_{\widetilde{z}}\right], 0\right), 0\right\} .
$$

From Definition 3, it is easy to obtain the following results [10]: 
(1) $0 \leq p(\widetilde{x} \geq \widetilde{z}) \leq 1$.

(2) $p(\widetilde{x} \geq \widetilde{z})+p(\widetilde{z} \geq \widetilde{x})=1$. Especially, $p(\widetilde{x} \geq \widetilde{x})=p(\widetilde{z} \geq \widetilde{z})=1 / 2$.

(3) $p(\widetilde{x} \geq \widetilde{z})=1$ if and only if $x^{L} \geq z^{U}$.

(4) $p(\widetilde{x} \geq \widetilde{z})=1 / 2$ if and only if $x^{L}+x^{U} \geq z^{L}+z^{U}$.

To rank the interval arguments $\widetilde{x}_{i}=\left[x_{i}^{L}, x_{i}^{U}\right](i=1,2, \cdots, n)$, we first compare each $\widetilde{x}_{i}$ with all $\tilde{x}_{j}(j=1,2, \cdots, n)$ by using (1). For convenience, we let $p_{i j}=p\left(\tilde{x}_{i} \geq \tilde{x}_{j}\right)$, and then construct a complementary matrix $P=\left(p_{i j}\right)_{n \times n^{\prime}}$ such that

$$
p_{i j} \geq 0, p_{i j}+p_{j i}=1, p_{i i}=1 / 2(i, j=1,2, \cdots, n) .
$$

Synthesizing the elements of each row of matrix $P$, we have

$$
p_{i}=\left(\sum_{j=1}^{n} p_{i j}+\frac{n}{2}-1\right) /(n(n-1))(i=1,2, \cdots, n) .
$$

Thus, we can rank arguments $\widetilde{x}_{i}(i=1,2, \cdots, n)$ in descending order in line with the value of $p_{i}(i=1,2, \cdots, n)$.

Remark: For the comparison between interval numbers, except for the possibility degree formula, some other formulas are also admirable, such as the admissible order [21].

\subsection{UGOWA Operator}

The OWA operator introduced by Yager [8] is a useful tool for aggregating the exact inputs that lie between the max and min operators. Since its introduction, the OWA operator has been studied by different authors. Inspired by the work of Yager, Zhou et al. [15] provided a new class of operator named as the UGOWA operator, which is an extension of UOWA operator. It can be defined as follows.

Definition 4. An UGOWA operator of dimension $n$ is a mapping UGOWA: $\Omega^{n} \rightarrow \Omega$ that has a weighting vector $W=\left(w_{1}, w_{2}, \cdots, w_{n}\right)^{T}$ with $w_{i} \in[0,1]$ and $\sum_{i=1}^{n} w_{i}=1$, such that:

$$
\operatorname{UGOWA}\left(\widetilde{x}_{1}, \widetilde{x}_{2}, \cdots \widetilde{x}_{n}\right)=\left(\sum_{i=1}^{n} w_{i} \widetilde{y}_{i}^{\lambda}\right)^{1 / \lambda}
$$

where $\widetilde{y}_{i}$ is the $i$ th largest of the $\widetilde{x}_{j}$, and $\lambda$ is a parameter such that $\lambda \in(-\infty, 0) \cup(0,+\infty)$.

If $\lambda=1$, then the UGOWA operator can degenerate to the UOWA operator.

If $\lambda \rightarrow 0$, then the UGOWA operator can become the UOWGA operator.

\subsection{HARA Utility Function}

A utility function $u(x)$ is a non-decreasing real valued function defined on the real numbers, which just captures the idea of aggregation operator that "an increase of any of the input values cannot decrease the output value". We investigate the HARA utility function [22,23]:

$$
\mu(x)=\frac{1-\gamma}{\beta \gamma}\left(\frac{\beta}{1-\gamma} x+\eta\right)^{\gamma} .
$$

with $\beta>0, \eta>0, \gamma \in(-\infty, 0) \cup(0,1)$. HARA utility is rather rich, since by suitable adjustments of the parameters one can obtain the power utility function $u(x)=x^{\gamma} / \gamma,(\beta=1-\gamma, \eta \rightarrow 0)$ and the exponential utility function $u(x)=-e^{-\beta x} / \beta,(\eta=1, \gamma \rightarrow-\infty)$ (for more details on parameters, one can refer to $[24,25])$. 


\section{Interval Generalized Weighted Utility Multiple Aggregation Operators}

Following [26-29], in this section, we will introduce a new aggregation operator by minimizing a new penalty function and obtain the IGOWUMA operator for the general utility function. Then, under the HARA utility function, we propose another new operator called the IGOWUMA-HARA operator.

\subsection{General Framework}

Let $\widetilde{x}_{i}=\left[\widetilde{x}_{i}^{L}, \widetilde{x}_{i}^{U}\right](i=1,2, \cdots, n)$ be a collection of interval arguments, and $W=\left(w_{1}, w_{2}, \cdots, w_{n}\right)^{T}$ be a weighting vector such that $w_{i} \in[0,1]$ and $\sum_{i=1}^{n} w_{i}=1$. We assume that the utility aggregation operator of dimension $n$ is a mapping $f$ determined by:

$$
\widetilde{z}=f\left(u\left(\widetilde{x}_{1}\right), u\left(\widetilde{x}_{2}\right), \cdots, u\left(\widetilde{x}_{n}\right)\right) .
$$

In the aggregation process, we hope that the smaller the deviation between the utility values $u\left(\widetilde{x}_{i}\right)(i=1,2, \cdots, n)$ and the aggregation result $\widetilde{z}$ is, the better effect of aggregation method shows.

Hence, to minimize the deviation between $\widetilde{z}$ and $u\left(\widetilde{x}_{i}\right)(i=1,2, \cdots, n)$, following $[26,29,30]$, we have:

$$
\min \widetilde{P}=\sum_{i=1}^{n} w_{i}\left(u^{\lambda}\left(\widetilde{x}_{i}\right) / u^{\lambda}(\widetilde{z})+u^{\lambda}(\widetilde{z}) / u^{\lambda}\left(\widetilde{x}_{i}\right)-2\right),
$$

where $\lambda$ is a parameter satisfying $\lambda \in(-\infty, 0) \cup(0,+\infty)$.

According to the necessary condition of extreme value, we can obtain:

$$
\widetilde{z}=u^{-1}\left[\left(\sum_{i=1}^{n} w_{i} u^{\lambda}\left(\widetilde{x}_{i}\right) / \sum_{i=1}^{n}\left(w_{i} / u^{\lambda}\left(\widetilde{x}_{i}\right)\right)\right)^{1 / 2 \lambda}\right] .
$$

Based on Equation (8), we can define an interval generalized weighted utility multiple averaging (IGWUMA) operator shown as follows.

Definition 5. Let $\widetilde{x}_{i}=\left[\widetilde{x}_{i}^{L}, \widetilde{x}_{i}^{U}\right](i=1,2, \cdots, n)$ be a collection of nonnegative interval numbers. An IGWUMA operator of dimension $n$ is a mapping IGWUMA: $\Omega^{n} \rightarrow \Omega$ such that:

$$
\operatorname{IGWUMA}\left(\widetilde{x}_{1}, \widetilde{x}_{2}, \cdots, \widetilde{x}_{n}\right)=u^{-1}\left[\left(\sum_{i=1}^{n} w_{i} u^{\lambda}\left(\widetilde{x}_{i}\right) / \sum_{i=1}^{n}\left(w_{i} / u^{\lambda}\left(\widetilde{x}_{i}\right)\right)\right)^{1 / 2 \lambda}\right],
$$

where the weight vector $W=\left(w_{1}, w_{2}, \cdots, w_{n}\right)^{T}$ satisfies $w_{i} \in[0,1]$ and $\sum_{i=1}^{n} w_{i}=1$, and $\lambda$ is a parameter such that $\lambda \in(-\infty, 0) \cup(0,+\infty)$.

If reordering the arguments in IGWUMA operator in descending order, we can obtain the IGOWUMA operator.

Definition 6. Let $\widetilde{x}_{i}=\left[\widetilde{x}_{i}^{L}, \widetilde{x}_{i}^{U}\right](i=1,2, \cdots, n)$ be a collection of nonnegative interval numbers. An IGOWUMA operator of dimension $n$ is a mapping IGOWUMA: $\Omega^{n} \rightarrow \Omega$ such that:

$$
\operatorname{IGOWUMA}\left(\widetilde{x}_{1}, \widetilde{x}_{2}, \cdots, \widetilde{x}_{n}\right)=u^{-1}\left[\left(\sum_{i=1}^{n} w_{i} u^{\lambda}\left(\widetilde{y}_{i}\right) / \sum_{i=1}^{n}\left(w_{i} / u^{\lambda}\left(\widetilde{y}_{i}\right)\right)\right)^{1 / 2 \lambda}\right],
$$

where $\lambda$ is a parameter such that $\lambda \in(-\infty, 0) \cup(0,+\infty), \widetilde{y}_{i}$ is the ith largest of $\widetilde{x}_{j}$, and all of $\widetilde{x}_{j}(j=1,2, \cdots, n)$ are interval numbers, which are compared with each other with the degree of 
possibility by Equations (1) and (3), and the weighting vector $W=\left(w_{1}, w_{2}, \cdots, w_{n}\right)^{T}$ satisfies $w_{i} \in[0,1]$ and $\sum_{i=1}^{n} w_{i}=1$.

IGOWUMA operator is idempotent, commutative, bounded, etc. These properties are shown as follows:

Property 1 (Idempotency). Let $F$ be the IGOWUMA operator. If $\widetilde{x}_{i}=\widetilde{x}$ for $i=1,2, \cdots, n$, then:

$$
F\left(\widetilde{x}_{1}, \widetilde{x}_{2}, \cdots, \widetilde{x}_{n}\right)=\widetilde{x}
$$

Property 2 (Commutativity). Let $F$ be the IGOWUMA operator. If $\widetilde{v}_{i}$ is any permutation of the arguments $\widetilde{x}_{i}$ for $i=1,2, \cdots, n$, then:

$$
F\left(\widetilde{x}_{1}, \widetilde{x}_{2}, \cdots, \widetilde{x}_{n}\right)=F\left(\widetilde{v}_{1}, \widetilde{v}_{2}, \cdots, \widetilde{v}_{n}\right)
$$

Property 3 (Boundedness). Let $F$ denote IGOWUM operator. If $\widetilde{t}_{1}=\left[t_{1}{ }^{L}, t_{1}{ }^{U}\right]=\left[\min _{1 \leq i \leq n} \alpha_{i}{ }^{L}, \min _{1 \leq i \leq n} \alpha_{i}{ }^{U}\right]$, $\widetilde{t}_{2}=\left[t_{2}{ }^{L}, t_{2}{ }^{U}\right]=\left[\max _{1 \leq i \leq n} \alpha_{i}{ }^{L}, \max _{1 \leq i \leq n} \alpha_{i}{ }^{U}\right]$, then:

$$
\widetilde{t}_{1} \leq F\left(\widetilde{\alpha}_{1}, \widetilde{\alpha}_{2}, \cdots \widetilde{\alpha}_{n}\right) \leq \widetilde{t}_{2}
$$

Proof. See Appendix A.

Property 4 (Monotonicity with respect to the degree of possibility). Let $F$ be the IGOWUMA operator, and $\widetilde{x}_{i}, \widetilde{z}_{i} \in \Omega$ for all $i$. If $p\left(\widetilde{x}_{i} \geq \widetilde{z}_{i}\right)=1$, then:

$$
p\left(F\left(\widetilde{x}_{1}, \widetilde{x}_{2}, \cdots, \widetilde{x}_{n}\right) \geq F\left(\widetilde{z}_{1}, \widetilde{z}_{2}, \cdots, \widetilde{z}_{n}\right)\right)=1 .
$$

Proof. See Appendix A.

\subsection{IGOWUMA-HARA Operator}

Under IGOWUMA operator and HARA utility function, we derive another interval aggregation operator called an IGOWUMA-HARA operator.

Definition 10. An IGOWUMA-HARA operator of dimension $\Omega^{n} \rightarrow \Omega$ is a mapping IGOWUMAHARA: $\Omega^{n} \rightarrow \Omega$ that has an associated weighting vector $W=\left(w_{1}, w_{2}, \cdots w_{n}\right)^{T}$ satisfying $w_{i} \in[0,1]$ and, such that:

$$
\operatorname{IGOWUMA}-\operatorname{HARA}\left(\widetilde{x}_{1}, \widetilde{x}_{2}, \cdots, \widetilde{x}_{n}\right)=\frac{1-\gamma}{\beta}\left[\left(\sum_{i=1}^{n} w_{i}\left(\frac{\beta}{1-\gamma} \widetilde{y}_{i}+\eta\right)^{\lambda \gamma} /\left(\sum_{i=1}^{n} w_{i} /\left(\frac{\beta}{1-\gamma} \widetilde{y}_{i}+\eta\right)^{\lambda \gamma}\right)\right)^{1 / 2 \lambda \gamma}-\eta\right]
$$

where $\lambda$ is a parameter such that $\lambda \in(-\infty, 0) \cup(0,+\infty)$, and $\widetilde{y}_{i}$ is the ith largest of $\widetilde{x}_{j}$, here, $\widetilde{x}_{j}(j=1,2, \cdots, n)$ are interval numbers, which are compared with each other by Equations (1) and (3).

Therefore, the IGOWUMA-HARA operator can be considered as the generalized mean of the interval generalized ordered weighted utility averaging-HARA (IGOWUA-HARA) operator and the interval generalized ordered weighted utility harmonic averaging-HARA (IGOWUHA-HARA) operator. That is to say, the IGOWUMA-HARA operator unifies IGOWUA-HARA operator, IGOWUHA-HARA operator and the geometric mean in the same formula considering the degree of importance that each operator has in the aggregation. 
Similar to Property 1 to Property 6, it is easy to find that IGOWUMA-HARA operator is idempotent, commutative, bounded, etc.

Table 1 shows that by taking different values of $\lambda, \beta, \eta, \gamma$ in the IGOWUMA-HARA operator, we can obtain different types aggregation operators such as the IGOWMA operator, the interval ordered weighted utility geometric averaging-HARA (IOWUGA-HARA) operator, IOWGA operator [10], the interval ordered weighted utility multiple averaging-HARA (IOWUMA-HARA) operator, the interval ordered weighted power utility multiple averaging (IPUMA) operator, the IOWMA operator, the interval linear ordered weighted geometric averaging (ILGA) operator, etc.

Table 1. Families of the interval generalized ordered weighted hyperbolic absolute risk aversion utility multiple averaging-HARA (IGOWUMA-HARA) operator.

\begin{tabular}{|c|c|c|}
\hline$\lambda$ & $\beta, \gamma, \eta$ & Formulation \\
\hline $\begin{array}{l}\lambda \in R \text { and } \\
\lambda \neq 0\end{array}$ & $\begin{array}{c}\beta=1-\gamma, \eta \rightarrow 0 \\
\gamma \rightarrow 1\end{array}$ & $\operatorname{IGOWMA}\left(\widetilde{x}_{1}, \widetilde{x}_{2}, \cdots, \widetilde{x}_{n}\right)=\left(\sum_{i=1}^{n} w_{i} \widetilde{y}_{i}^{\lambda} /\left(\sum_{i=1}^{n} w_{i} / \widetilde{y}_{i}^{\lambda}\right)\right)^{1 / 2 \lambda}$ \\
\hline \multirow{2}{*}{$\lambda \rightarrow 0$} & $\begin{array}{c}\beta>0, \eta>0 \\
\gamma \in R^{-} \text {and } \gamma \in(0,1)\end{array}$ & IOWUGA - HARA $\left(\widetilde{x}_{1}, \widetilde{x}_{2}, \cdots, \widetilde{x}_{n}\right)=\frac{1-\gamma}{\beta}\left(\prod_{i=1}^{n}\left(\frac{\beta}{1-\gamma} \widetilde{y}_{i}+\eta\right)^{w_{i}}-\eta\right)$ \\
\hline & $\begin{array}{l}\beta>0, \gamma \in R^{-} \text {and } \\
\gamma \in(0,1), \eta \rightarrow 0\end{array}$ & $\operatorname{IOWGA}\left(\widetilde{x}_{1}, \widetilde{x}_{2}, \cdots, \widetilde{x}_{n}\right)=\prod_{i=1}^{n} \widetilde{y}_{i}^{w_{i}}$ \\
\hline \multirow{5}{*}{$\begin{array}{l}\lambda=1 \\
\quad \text { or } \\
\lambda=-1\end{array}$} & $\begin{array}{c}\beta>0, \eta>0 \\
\gamma \in R^{-} \text {and } \gamma \in(0,1)\end{array}$ & $\operatorname{IOWUMA}-\operatorname{HARA}\left(\widetilde{x}_{1}, \widetilde{x}_{2}, \cdots, \widetilde{x}_{n}\right)=\frac{1-\gamma}{\beta}\left(\left(\frac{\sum_{i=1}^{n} w_{i}\left(\frac{\beta}{1-\gamma} \widetilde{y}_{i}+\eta\right)^{\gamma}}{\sum_{i=1}^{n} w_{i} /\left(\frac{\beta}{1-\gamma} \widetilde{y}_{i}+\eta\right)^{\gamma}}\right)^{1 / 2 \gamma}-\eta\right.$ \\
\hline & $\begin{array}{l}\beta>0, \gamma \in R^{-} \text {and } \\
\gamma \in(0,1), \eta \rightarrow 0\end{array}$ & $\operatorname{IPUMA}\left(\widetilde{x}_{1}, \widetilde{x}_{2}, \cdots, \widetilde{x}_{n}\right)=\left(\sum_{i=1}^{n} w_{i} \widetilde{y}_{i}^{\gamma} / \sum_{i=1}^{n} w_{i} / \widetilde{y}_{i}^{\gamma}\right)^{1 / 2 \gamma}$ \\
\hline & $\begin{array}{c}\beta>0, \eta \rightarrow 0 \\
\gamma \rightarrow 1\end{array}$ & $\operatorname{IOWMA}\left(\widetilde{x}_{1}, \widetilde{x}_{2}, \cdots, \widetilde{x}_{n}\right)=\left(\sum_{i=1}^{n} w_{i} \widetilde{y}_{i} / \sum_{i=1}^{n} w_{i} / \widetilde{y}_{i}\right)^{1 / 2}$ \\
\hline & $\begin{array}{l}\beta>0, \eta>0 \\
\quad \gamma \rightarrow 0\end{array}$ & $\operatorname{ILGA}\left(\widetilde{x}_{1}, \widetilde{x}_{2}, \cdots, \widetilde{x}_{n}\right)=\frac{1}{\beta}\left(\prod_{i=1}^{n}\left(\beta \widetilde{y}_{i}+\eta\right)^{w_{i}}-\eta\right)$ \\
\hline & $\begin{aligned} & \beta>0, \gamma \rightarrow 0 \\
& \quad \eta \rightarrow 0\end{aligned}$ & $\operatorname{IOWGA}\left(\widetilde{x}_{1}, \widetilde{x}_{2}, \cdots, \widetilde{x}_{n}\right)=\prod_{i=1}^{n} \widetilde{y}_{i}^{w_{i}}$ \\
\hline
\end{tabular}

IGOWMA: interval generalized ordered weighted multiple averaging; IOWUG-HARA: interval ordered weighted utility geometric averaging-HARA; IOWGA: interval ordered weighted geometric averaging; IOWUM-HARA: interval ordered weighted utility multiple averaging-HARA; IPUMA: interval ordered weighted power utility multiple averaging; IOWMA: interval ordered weighted multiple averaging; ILGA: interval linear ordered weighted geometric averaging.

\section{Weights Determination}

In order to determine the weights of the IGOWUMA-HARA operator, we propose the concept of similarity degree of interval numbers and analyze its properties. We further provide a new optimization model, which can obtain an optimal weight vector for the IGOWUMA-HARA operator.

In the literature [10], Xu presented a weighting model of DUOWA operator by applying the absolute deviation measure and the optimism degree. Actually, as far as measuring deviation, the relative deviation measure is more effective than the absolute deviation measure. Thus, Zhou et al. [15] developed a new model to determine the weights of UGOWA operator via the relative deviation measure. However, the weighted models do not provide a uniform weighting vector in the literature $[10,15]$, which will affect the accuracy of the decision results. Therefore, we will present a new preemptive nonlinear objective programming model to determining the IGOWUMA-HARA operator weights, which can provide a uniform weighting vector to guarantee the uniform standard comparison between the alternatives and reduce the influence of unfair arguments on the aggregated results.

Let $X=\left\{x_{1}, x_{2}, \cdots, x_{m}\right\}$ be a finite set of alternatives, $C=\left\{c_{1}, c_{2}, \cdots, c_{n}\right\}$ a finite set of attributes, $\widetilde{B}=\left(\widetilde{b}_{i j}\right)_{m \times n}$ is the interval decision matrix, where $\widetilde{b}_{i j}=\left[b_{i j}^{L}, b_{i j}^{U}\right] \in \Omega$ is a preference value, which 
takes the form of interval argument, given by the decision maker for the alternative $x_{i} \in X$ with regard to the attribute $c_{j} \in C$. Assume that the partial weight information of attributes is:

$$
H=\left\{\left(w_{1}, w_{2}, \cdots, w_{n}\right)^{T} \mid \rho_{j}^{L} \leq w_{j} \leq \rho_{j}^{U}, j=1,2, \cdots, n, \sum_{j=1}^{n} \rho_{j}^{L} \leq 1, \sum_{j=1}^{n} \rho_{j}^{U} \geq 1\right\} .
$$

Standardize matrix $\widetilde{B}=\left(\widetilde{b}_{i j}\right)_{m \times n}$ into matrix $\widetilde{R}=\left(\widetilde{r}_{i j}\right)_{m \times n}=\left(\left[r_{i j}^{L}, r_{i j}^{U}\right]\right)_{m \times n^{\prime}}$ then the integrated attribute value, which is obtained by using the aggregation operator IGOWUMA-HARA to gather the attribute preference information value of each alternative, is still the interval number $\widetilde{z}_{i}(W)=\left[z_{i}^{L}(W), z_{i}{ }^{U}(W)\right](i=1,2, \cdots, m)$, where:

$$
\begin{aligned}
& z_{i}^{L}(W)=\frac{1-\gamma}{\beta}\left[\left(\sum_{j=1}^{n} w_{j}\left(\frac{\beta}{1-\gamma} y_{i j}^{L}+\eta\right)^{\lambda \gamma} /\left(\sum_{j=1}^{n} w_{j} /\left(\frac{\beta}{1-\gamma} y_{i j}^{L}+\eta\right)^{\lambda \gamma}\right)\right)^{1 / 2 \lambda \gamma}-\eta\right](i=1,2, \cdots, m), \\
& z_{i}^{U}(W)=\frac{1-\gamma}{\beta}\left[\left(\sum_{j=1}^{n} w_{j}\left(\frac{\beta}{1-\gamma} y_{i j}^{U}+\eta\right)^{\lambda \gamma} /\left(\sum_{j=1}^{n} w_{j} /\left(\frac{\beta}{1-\gamma} y_{i j}^{U}+\eta\right)^{\lambda \gamma}\right)\right)^{1 / 2 \lambda \gamma}-\eta\right](i=, 1,2, \cdots, m) .
\end{aligned}
$$

In order to calculate a uniform weighting vector to guarantee the uniform standard comparison between the alternatives and measure their fair competition under the condition of valid comparison between various alternatives. Following [31], we can construct the nonlinear programming model as follows:

$$
\begin{aligned}
& \min \sum_{j=1}^{n} w_{j}\left(\left(\frac{y_{i j}}{z_{i}^{L}}\right)^{\lambda}+\left(\frac{z_{i}^{L}}{y_{i j}}\right)^{\lambda}-2\right)+\sum_{j=1}^{n-1}\left(\left(\frac{w_{j}}{w_{j+1}}\right)^{\lambda}+\left(\frac{w_{j+1}}{w_{j}}\right)^{\lambda}-2\right)(i=1,2, \cdots, m) \\
& \min \sum_{j=1}^{n} w_{j}\left(\left(\frac{y_{i j}}{z_{i}^{U}}\right)^{\lambda}+\left(\frac{z_{i}^{U}}{y_{i j}}\right)^{\lambda}-2\right)+\sum_{j=1}^{n-1}\left(\left(\frac{w_{j}}{w_{j+1}}\right)^{\lambda}+\left(\frac{w_{j+1}}{w_{j}}\right)^{\lambda}-2\right)(i=1,2, \cdots, m) \\
& \text { s.t. } w_{j} \in H \quad j=1,2, \cdots, n \\
& \sum_{j=1}^{n} w_{j}=1
\end{aligned}
$$

where $H$ is partial weight information.

Model (19) can not only determine the uniform weight vector, making the alternative is comparable, but also can ensure that the alternatives can compete in a fair environment. As can be seen from the model (19), the expected value of the objective function $z_{1}(W)$ is $\sum_{i=1}^{m}\left\{\frac{1-\gamma}{\beta}\left[\left(\sum_{j=1}^{n} \rho_{j}^{L}\left(\frac{\beta}{1-\gamma} y_{i j}^{L}+\eta\right)^{\lambda \gamma} /\left(\sum_{j=1}^{n} \rho_{j}^{L} /\left(\frac{\beta}{1-\gamma} y_{i j}^{L}+\eta\right)^{\lambda \gamma}\right)\right)^{1 / 2 \lambda \gamma}-\eta\right]\right\}$ while expected value of the objective function $z_{2}(W)$ is $\sum_{i=1}^{m}\left\{\frac{1-\gamma}{\beta}\left[\left(\sum_{j=1}^{n} \rho_{j}^{U}\left(\frac{\beta}{1-\gamma} y_{i j}^{U}+\eta\right)^{\lambda \gamma} /\left(\sum_{j=1}^{n} \rho_{j}^{U} /\left(\frac{\beta}{1-\gamma} y_{i j}^{U}+\eta\right)^{\lambda \gamma}\right)\right)^{1 / 2 \lambda \gamma}-\eta\right]\right\}$.

In order to solve the multi-objective programming model, the model (19) can be transformed into the following model:

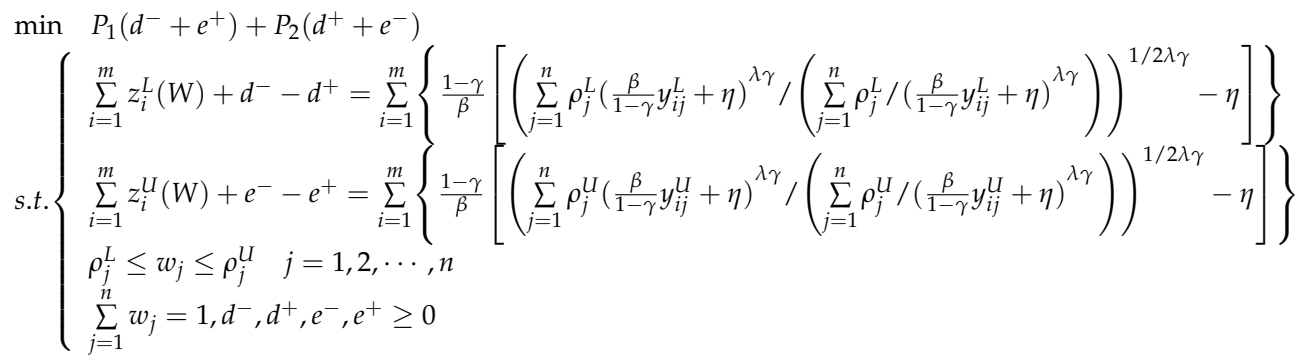


where $P_{1}, P_{2}$ are the priority factor which indicates the relative importance of each target; $d^{-}, d^{+}$are the lower and upper deviation variables of the objective function $\sum_{i=1}^{m} z_{i}^{L}(W)$ which is lower or higher than the expected value $\sum_{i=1}^{m}\left\{\frac{1-\gamma}{\beta}\left[\left(\sum_{j=1}^{n} \rho_{j}^{L}\left(\frac{\beta}{1-\gamma} y_{i j}^{L}+\eta\right)^{\lambda \gamma} /\left(\sum_{j=1}^{n} \rho_{j}^{L} /\left(\frac{\beta}{1-\gamma} y_{i j}^{L}+\eta\right)^{\lambda \gamma}\right)\right)^{1 / 2 \lambda \gamma}-\eta\right]\right\}$, respectively; $e^{-}, e^{+}$ are the lower and upper deviation variables of the objective function $\sum_{i=1}^{m} z_{i}^{U}(W)$ which is lower or higher than the expected value $\sum_{i=1}^{m}\left\{\frac{1-\gamma}{\beta}\left[\left(\sum_{j=1}^{n} \rho_{j}^{U}\left(\frac{\beta}{1-\gamma} y_{i j}^{U}+\eta\right)^{\lambda \gamma} /\left(\sum_{j=1}^{n} \rho_{j}^{U} /\left(\frac{\beta}{1-\gamma} y_{i j}^{U}+\eta\right)^{\lambda \gamma}\right)\right)^{1 / 2 \lambda \gamma}-\eta\right]\right\}$, respectively.

\section{An Approach to IMAGDM Based on the IGOWUMA-HARA Operator}

This section develops a new approach for MAGDM based on the IGOWUMA-HARA operator.

For a MAGDM problem, let $X=\left\{x_{1}, x_{2}, \cdots, x_{m}\right\}$ be a finite set of alternatives, $C=\left\{c_{1}, c_{2}, \cdots, c_{n}\right\}$ be a finite set of attributes and $V^{(k)}=\left(v_{1}^{(k)}, v_{2}^{(k)}, \cdots, v_{n}^{(k)}\right)^{T}$ be the vector of attributes such that $v_{j}^{(k)} \geq 0$ and $\sum_{j=1}^{n} v_{j}^{(k)}=1 . D=\left\{d_{1}, d_{2}, \cdots, d_{l}\right\}$ is a finite set of DMs and $W=\left(w_{1}, w_{2}, \cdots, w_{l}\right)^{T}$ is the vector of DMs satisfying $w_{k} \geq 0$ and $\sum_{k=1}^{l} w_{k}=1$. In addition, assume that $\widetilde{B}^{(k)}=\left(\widetilde{b}_{i j}^{(k)}\right)_{m \times n}$ is the interval decision matrix, where $\widetilde{b}_{i j}^{(k)}=\left[b_{i j}^{(k) L}, b_{i j}^{(k) U}\right] \in \Omega$ is a preference value, which takes the form of interval argument, given by the decision maker $d_{k} \in D$ for the alternative $x_{i} \in X$ with regard to the criterion $c_{j} \in C$.

Since different attributes have different measurement scales in MAGDM problem, it is necessary for the standardization of attributes to avoid the variance among different attributes. In this paper, we consider two attributes, i.e., profit type and cost type. Let $I_{1}$ be a set of benefit attributes and $I_{2}$ be a set of cost attribute. The decision matrix $\widetilde{B}^{(k)}$ can be transformed into a corresponding decision matrix $\widetilde{R}^{(k)}$ via the following formulas [31]:

$$
\begin{aligned}
& \left\{\begin{array}{l}
r_{i j}^{(k) L}=b_{i j}^{(k) L} / \sqrt{\sum_{i=1}^{m}\left(b_{i j}^{(k) U}\right)^{2}}, \\
r_{i j}^{(k) U}=b_{i j}^{(k) U} / \sqrt{\sum_{i=1}^{m}\left(b_{i j}^{(k) L}\right)^{2}}, j \in I_{1}, \quad i=1,2, \cdots, m .
\end{array}\right. \\
& \left\{\begin{array}{l}
r_{i j}^{(k) L}=1 / b_{i j}^{(k) U} / \sqrt{\sum_{i=1}^{m} 1 /\left(b_{i j}^{(k) L}\right)^{2}}, \\
r_{i j}^{(k) U}=1 / b_{i j}^{(k) L} / \sqrt{\sum_{i=1}^{m} 1 /\left(b_{i j}^{(k) U}\right)^{2}},
\end{array} \in I_{2}, \quad i=1,2, \cdots, m .\right.
\end{aligned}
$$

Based on the above explanation, we develop an approach to MAGDM problem. The concrete steps are shown as follows.

Step 1. Standardize the decision matrixes.

Based on Equations (21) and (22), the matrixes $\widetilde{B}^{(k)}=\left(\widetilde{b}_{i j}^{(k)}\right)_{m \times n}(k=1,2, \cdots, l)$ can be transformed into standardization matrixes $\widetilde{R}^{(k)}=\left(\widetilde{r}_{i j}^{(k)}\right)_{m \times n}(k=1,2, \cdots, l)$.

Step 2. Calculate the weight vector of attributes.

Based on model (19), the IGOWUMA-HARA weight vector can be derived: $V^{(k)}=\left(v_{1}^{(k)}, v_{2}^{(k)}, \ldots, v_{n}^{(k)}\right)^{T}$ is the vector of attributes such that $v_{j}^{(k)} \geq 0$ and $\sum_{j=1}^{n} v_{j}^{(k)}=1$.

Step 3. Aggregate the decision matrixes into a collective decision matrix. 


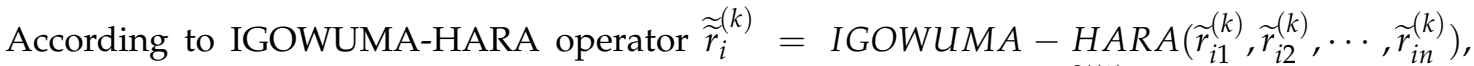
$i=1,2, \cdots, m, k=1,2, \cdots, l$, we can aggregate all the decision matrices $\widetilde{R}^{(k)}(k=1,2, \cdots, l)$ into a collective decision matrix $\widetilde{\widetilde{R}}=\left(\widetilde{\widetilde{r}}_{i j}\right)_{m \times l}$.

Step 4. Calculate the weight vector of the DMs.

Based on model (19), the weight vector of decision makers can be calculated $W=\left(w_{1}, w_{2}, \cdots, w_{l}\right)^{T}$, where $w_{k} \geq 0(k=1,2, \cdots, l)$ and $\sum_{k=1}^{l} w_{k}=1$.

Step 5. Aggregate the collective overall the interval preference value.

According to IGOWUMA-HARA operator $\widetilde{t}_{i}=\operatorname{IGOWUMA}-\operatorname{HARA}\left(\widetilde{\widetilde{r}}_{11}, \widetilde{\widetilde{r}}_{i 2}, \cdots, \widetilde{\widetilde{r}}_{i l}\right)$, $i=1,2, \cdots, m$, we can obtain the collective overall preference value $\widetilde{t}_{i}$ of the alternative $x_{i}$.

Step 6. Compare each interval argument $\widetilde{t}_{i}$ with all $\widetilde{t}_{i}$ by Equation (1) and get the complementary matrix $P=\left(p_{i j}\right)_{m \times m}$.

Step 7. Synthesize all elements of matrix $P=\left(p_{i j}\right)_{m \times m}$ by Equation (3) and get $p_{i}(i=1,2, \cdots, m)$.

Step 8. Rank the collective overall preference values $\widetilde{t}_{j}$ in descending order in accordance with the values of $p_{i}(i=1,2, \cdots, m)$.

Step 9. Select the best alternative.

Rank all the alternatives $x_{i}(i=1,2, \cdots, m)$ in descending order and consequently select the best one in the light of the collective overall preference value $\widetilde{t}_{i}(i=1,2, \cdots, m)$.

\section{Illustrative Example}

In this section, a practical group decision making problem involving the prioritization of a set of information technology improvement projects adapted from $\mathrm{Xu}$ [10] is used to illustrate the proposed uncertain aggregation operator.

The steering committee of information management department of Midwest American's Manufacturing Corp must consider expanding and implementing a set of six projects preferentially. The projects, $x_{1}$ quality management information, $x_{2}$ inventory control, $x_{3}$ customer order tracking, $x_{4}$ materials purchasing management, $x_{5}$ fleet management and $x_{6}$ design change management, are proposed by different regional managers, aiming at improving information and technology. What the committee is concerned about is the projects' prioritized potential contribution, accordingly, from the highest level to the lowest. At a strategic level, company acquires competitive advantages over its rivals in the industry. In evaluating the potential contribution of each project, three factors are taken into consideration, namely, $c_{1}$-productivity, $c_{2}$-differentiation, and $c_{3}$-management.

Suppose that the decision experts of the investment company are four persons $d_{1}, d_{2}, d_{3}$ and $d_{4}$, they offer their own opinions with scores ranging from 0 to 100 , over the projects $x_{i}(i=1,2, \cdots, 6)$ with respect to the factors $c_{j}(j=1,2,3)$, and the results are shown as in Tables A1-A4 (See Appendix B).

In addition, for simplicity, without loss of generality, we assume that the parameters of the IGOWUMA-HARA operator are respectively: $\lambda=1, \beta=3, \eta=1$ and $\gamma=0.1$.

\subsection{Computational Results}

Based on the above information and by applying the software of Lingo 11.0 developed by American Lindo systems, we can use the proposed decision-making approach to ranking the order of enterprises. The concrete steps are shown as follows.

Step 1. Suppose that $\widetilde{R}^{(k)}=\left(\widetilde{r}_{i j}^{(k)}\right)_{6 \times 3}=\widetilde{B}^{(k)}(k=1,2,3,4)$. Based on model (19), the optimal weighting vector of attributes with regard to the $k$ th $\mathrm{DM} d_{k}(k=1,2,3,4)$ is obtained as follows:

$$
\begin{aligned}
& V_{1}=(0.3333,0.5000,0.1667)^{T}, V_{2}=(0.4852,0.3000,0.2148)^{T}, \\
& V_{3}=(0.4270,0.3000,0.2730)^{T}, V_{4}=(0.3556,0.4262,0.2182)^{T} .
\end{aligned}
$$




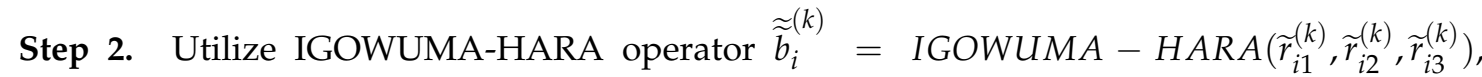
$(i=1,2, \ldots, 6 ; k=1,2,3,4)$ to aggregate the uncertain variables of the decision matrix $\widetilde{R}^{(k)}=\left(\widetilde{r}_{i j}^{(k)}\right)_{m \times n}(k=1,2,3,4)$ and get the individual overall preference value $\widetilde{b}_{i}^{(k)}$ corresponding to the alternative the alternative $x_{i}$ given by $d_{k}$ :

$$
\begin{aligned}
& \widetilde{b}_{1}^{(1)}=[85.2612,93.0251], \quad \widetilde{b}_{2}^{(1)}=[70.2762,82.2614], \quad \widetilde{b}_{3}^{(1)}=[78.2466,87.8873], \\
& \widetilde{b}_{4}^{(1)}=[74.1237,84.8533], \quad \widetilde{b}_{5}^{(1)}=[83.3855,89.2769], \quad \widetilde{b}_{6}^{(1)}=[73.4909,82.2516], \\
& \widetilde{b}_{1}^{(2)}=[73.0026,83.4971], \quad \widetilde{b}_{2}^{(2)}=[84.1709,90.2273], \quad \widetilde{b}_{3}^{(2)}=[81.1823,91.8827], \\
& \widetilde{b}_{4}^{(2)}=[85.2105,90.2273], \quad \widetilde{b}_{5}^{(2)}=[83.9638,88.9699], \quad \widetilde{b}_{6}^{(2)}=[80.4767,90.2273], \\
& \widetilde{b}_{1}^{(3)}=[86.4702,93.6081], \quad \widetilde{b}_{2}^{(3)}=[78.5526,85.6307], \quad \widetilde{b}_{3}^{(3)}=[83.6048,90.6761], \\
& \widetilde{b}_{4}^{(3)}=[85.5306,95.6810], \quad \widetilde{b}_{5}^{(3)}=[85.0510,92.3882], \quad \widetilde{b}_{6}^{(3)}=[76.4430,84.6055], \\
& \widetilde{b}_{1}^{(4)}=[84.1194,90.2458], \quad \widetilde{b}_{2}^{(4)}=[82.9530,92.9680], \quad \widetilde{b}_{3}^{(4)}=[87.0965,93.8859], \\
& \widetilde{b}_{4}^{(4)}=[83.8715,89.8769], \quad \widetilde{b}_{5}^{(4)}=[84.4089,89.4197], \quad \widetilde{b}_{6}^{(4)}=[85.9521,92.0983] \text {. }
\end{aligned}
$$

We further can derive a collective decision matrix $\widetilde{B}=\left(\widetilde{b}_{i k}\right)_{6 \times 4}$.

Step 3. Apply model (19) to calculate the optimal weighting vector of four DMs:

$$
W=(0.2924,0.2076,0.1000,0.4000)^{T}
$$

Step 4. Utilize the collective decision information given by matrix $\widetilde{B}=\left(\widetilde{b}_{i k}\right)_{6 \times 4}$ and calculate the collective overall preference value $\widetilde{t}_{i}=\operatorname{IGOWUMA}-\operatorname{HARA}\left(\widetilde{b}_{i 1}, \widetilde{b}_{i 2}, \widetilde{b}_{i 3}, \widetilde{b}_{i 4}\right)$ of the alternative $x_{i}(i=1,2, \ldots, 6)$ :

$$
\begin{array}{lll}
\widetilde{t}_{1}=[82.2296,89.9208], & \widetilde{t}_{2}=[78.8360,88.4159], & \widetilde{t}_{3}=[82.8474,91.3612], \\
\widetilde{t}_{4}=[81.3218,89.0049], & \widetilde{t}_{5}=[84.0798,89.5765], & \widetilde{t}_{6}=[80.0468,87.9750] .
\end{array}
$$

Step 5. Compare each interval argument $\widetilde{t}_{i}$ with all $\widetilde{t}_{z}(z=1,2, \ldots, 6)$ by Equation (1) and get the complementary matrix $P=\left(p_{i z}\right)_{6 \times 6}$ :

$$
P=\left[\begin{array}{llllll}
0.5000 & 0.6418 & 0.4365 & 0.5593 & 0.4429 & 0.6322 \\
0.3582 & 0.5000 & 0.3078 & 0.4109 & 0.2876 & 0.4780 \\
0.5635 & 0.6922 & 0.5000 & 0.6198 & 0.5197 & 0.6881 \\
0.4407 & 0.5891 & 0.3802 & 0.5000 & 0.3737 & 0.5738 \\
0.5571 & 0.7124 & 0.4803 & 0.6263 & 0.5000 & 0.7099 \\
0.3678 & 0.5220 & 0.3119 & 0.4262 & 0.2901 & 0.5000
\end{array}\right]
$$

Step 6. Synthesize all elements in each line of the matrix $P=\left(p_{i z}\right)_{6 \times 6}$ by Equation (3) to get $p_{1}=0.1738, p_{2}=0.1448, p_{3}=0.1861, p_{4}=0.1619, p_{5}=0.1862, p_{6}=0.1473$.

Step 7. Rank the collective overall preference values $\widetilde{t}_{i}(i=1,2, \ldots, m)$ in descending order in the light of the values of $p_{i}(i=1,2, \cdots, m)$.

$$
p_{5}>p_{3}>p_{1}>p_{4}>p_{6}>p_{2}
$$

Step 8. Rank all the alternatives $x_{i}(i=1,2, \cdots, 6)$ in accordance with the collective overall preference values $\widetilde{t}_{i}(i=1,2, \cdots, 6)$ :

$$
x_{5} \succ x_{3} \succ x_{1} \succ x_{4} \succ x_{6} \succ x_{2}
$$

Thus, the best investment alterative is fleet management $x_{5}$. 


\subsection{Sensitive Analysis}

Sensitivity analysis is the investigation of some potential changes and errors of rating values and their impact on the final ranking order [32]. In this paper, some sensitivity analyses are conducted to investigate the impact of changing the values of parameters $\beta, \gamma, \eta$ in IGOWUMA-HARA operator on the alternatives' ranking order.

Based on the parameters given by Section 6.1, Table A5 (See Appendix B) shows the impact of changing the parameters of IGOWUMA-HARA operator on the best investment alternative, including the changing of single parameter (No. 1 to No. 15), two parameters (No. 16 to No. 30), three parameters (No. 31 to No. 35), respectively.

From Table A5, we find that the selection of the best alternative will change with the single parameter $\gamma$ (or $\eta$ ) decreasing or with the parameter $\beta$ increasing to a certain point (See, No. 1 to No. 15). In other words, the changing of single parameter has an influence on the selection of the best alternative.

For example, the best alternative will change from $x_{5}$ to $x_{3}$ when the parameter $\gamma$ (resp. $\eta$ ) decreases from 0.9 to 0.1 (resp. from 3 to 1) (See, No. 1 to No. 10), while this change will happen as the parameter $\beta$ increases from 0.1 to 3 (See, No. 11 to No. 15). Moreover, the absolute risk aversion coefficient $r(x, \gamma, \beta, \eta)$ will decrease with $\gamma$ or $\eta$ increases, while it will increase with the rise of $\beta$ (See, No. 1 to No. 15). This can be explained by the first partial derivative of the absolute risk aversion coefficient. Note that the first partial derivative of $r(x, \gamma, \beta, \eta)$ for HARA utility is $r_{\gamma}^{\prime}(x, \gamma, \beta, \eta)=-\beta^{2} x /(\beta x+\eta(1-\gamma))^{2} \leq 0, r_{\eta}^{\prime}(x, \gamma, \beta, \eta)=-\beta(1-\gamma)^{2} /(\beta x+\eta(1-\gamma))^{2}<0$ and $r_{\beta}^{\prime}(x, \gamma, \beta, \eta)=\eta(1-\gamma)^{2} /(\beta x+\eta(1-\gamma))^{2}>0$, respectively. Then, we can conclude that $r(x, \gamma, \beta, \eta)$ will decrease with the increasing of $\gamma$ or $\eta$, while it will increase with the increasing of $\beta$.

In addition, in Table A5, we can see that the changing of two parameters (or three parameters) will also affect the selection of the best alternative. In particular, the best alternative will change with the absolute risk aversion coefficient $r(x, \gamma, \beta, \eta)$ increasing to a certain point, and this changing tendency is also suitable for the single parameter case.

For example, under the condition of single parameter $\gamma$, the best alternative will change from $x_{5}$ to $x_{3}$ when the absolute risk aversion coefficient $r(x, \gamma, \beta, \eta)$ increases from 0.353 to 1.50 (See, No. 1 to No. 5). For the case of two parameters $\gamma$ and $\eta$, the best alternative will change from $x_{5}$ to $x_{3}$ when $r(x, \gamma, \beta, \eta)$ increases from 0.286 to 2.093 (See, No. 21 to No. 25). Under the changing of three parameters, the best alternative will change from $x_{5}$ to $x_{3}$ when $r(x, \gamma, \beta, \eta)$ increases from 0.264 to 1.448 (See, No. 31 to No. 35).

This situation can be understood by the meaning of $r(x, \gamma, \beta, \eta)$. Notice that with the absolute risk aversion coefficient increasing, the risk attitude of DM's involved in the evaluation of decision-making information will become more prudent. In other words, the higher the risk aversion is, the more conservative the DM is. From Tables A1-A4, we find that the alternative $x_{3}$ is better than the alternative $x_{5}$ with respect to the attributes $c_{1}$ and $c_{3}$. Recall that $c_{1}$ and $c_{3}$ respectively denote the productivity and the management. These two attributes, for the information technology improvement projects in reality, draw more attention than those of $c_{1}$ (productivity) and $c_{3}$ (management). Therefore, from the viewpoint of information technology improvement efficiency, the alternative $x_{3}$ is superior to the alternative $x_{5}$ with the increasing of $r(x, \gamma, \beta, \eta)$.

In addition, Table A5 shows that no matter what the changing of parameter (single parameter, two or three parameters) is, the best alternative is still $x_{5}$ if the value of $r(x, \gamma, \beta, \eta)$ is less than or equal to 0.945 , while it will become $x_{3}$ when the value of $r(x, \gamma, \beta, \eta)$ is greater than 0.945 . Here, we should note that the point of $r(x, \gamma, \beta, \eta)$ equal to 0.945 is only suitable for this example because the calculation of the best investment alternative involves other factors such as the values of attributes, weights of attributes, the relatively important weight of DMs and the relatively important degree of total deviation. 


\subsection{Comparative Analysis of the Obtained Results}

What needs to be mentioned is that Zhou et al. [15] also adapted the same illustrative example to illustrate the developed UGOWA operator. Therefore, we mainly compare the ranking results got by the DUOWA operator, UGOWA operator and the proposed IGOWUMA-HARA operator.

The comparison results are shown in Table 2. It can be seen that the best alternative is the same, which explains the applicability of the proposed method. However, the rankings are not completely consistent in [10], [15] and this paper. This difference implies that our utility aggregation operator is superior to the DUOWA operator [10] and UGOWA operator [15], which can be explained by the following analysis.

Table 2. The comparison results of the two operators.

\begin{tabular}{ccl}
\hline Operator & Score & Ranking \\
\hline The DUOWA operator & $(3.0320,1.9227,3.4656,3.4102,3.5927,2.5769)$ & $x_{5} \succ x_{3} \succ x_{4} \succ x_{1} \succ x_{6} \succ x_{2}$ \\
The UGOWA operator & $(0.1562,0.1317,0.1802,0.1768,0.1814,0.1738)$ & $x_{5} \succ x_{3} \succ x_{4} \succ x_{6} \succ x_{1} \succ x_{2}$ \\
The proposed operator & $(0.1738,0.1448,0.1861,0.1619,0.1862,0.1473)$ & $x_{5} \succ x_{3} \succ x_{1} \succ x_{4} \succ x_{6} \succ x_{2}$ \\
\hline
\end{tabular}

(a) Theoretically, both of the DUOWA operator and UGOWA operator use the mean to eliminate the difference of variable values and lead to the deviation between the variable values and the results of variable aggregation, while the presented aggregation operator in this paper just modifies this limitation.

For instance, the DUOWA operator and UGOWA operator cannot precisely depict the deviation between the interval variable values and the aggregation results of interval variable because when aggregating the interval variables, they directly use the mean to eliminate the difference of data, which leads to the deviation between the aggregation values and the result of aggregation. However, the presented aggregation operator IGOWUMA-HARA can perfectly depict the deviation between the interval variable values and the aggregation results of interval variable via penalty function theory, which can eliminate the difference of data as possible and minimize the deviation between the interval variable values and the results of interval variable aggregation. Hence, it can effectively reduce the loss and distortion of interval information in aggregating process, and correspondingly improve the precision of the decision-making results.

(b) When assessing the alternatives, the DUOWA operator and UGOWA operator just consider the objective interval arguments, and the associated weights only depend on the aggregated interval arguments, but did not provide a uniform weighting vector based on the degree of similarity to compare the alternatives. On the contrary, the proposed aggregation operator sufficiently considers the subjective risk attitude of DMs by using utility function, and based on the developed similarity measure of preference values, the weighting vector of attributes is reached by an optimization model. The weighting vector cannot only guarantee that the alternatives can be contrasted according to the unified standard, but also can insure the theoretical foundations of effectiveness of proposed method.

For example, we see that the ranking result is always $x_{4} \succ x_{1}$ by using the presented method in $[10,15]$. However, the ranking result is $x_{1} \succ x_{4}(\lambda=1, \beta=3, \eta=1$ and $\gamma=0.1)$ when utilizing the developed method in this paper. This situation can be explained by the fact that the alternative $x_{1}$ is better than the alternative $x_{4}$ with respect to the attributes $c_{1}$ and $c_{2}$ from Tables A1-A4. Recall that $c_{1}$ denote the productivity, which is provided to assess the potential benefits of a proposed project, and ultimately, to increase the effectiveness and efficiency of the firm's manufacturing and service operations; and $c_{2}$ denote the differentiation factor, which is introduced to assess the potential of a proposed project, in this way, the firm's products and services can be distinguished from its competitors fundamentally, making itself more desirable to its customers. These two attributes, for the information technology improvement projects in reality, draw more attention than those of $c_{1}$ (productivity) and $c_{2}$ 
(differentiation). Therefore, from the viewpoint of information technology improvement efficiency, the alternative $x_{1}$ is superior to the alternative $x_{4}$ with the increasing of $r(x, \gamma, \beta, \eta)$.

(c) The presented method is widely used in the real decision-making process, and the decision-making based on utility operator is more in accordance with $\mathrm{DMs}^{\prime}$ actual decision-making behavior. The results show that (i) the utility values obtained by the proposed method seem more similar to their original attribute values; (ii) the objective is to understand the subjective feelings of DMs by using developed method. However, the traditional method cannot have the above characteristics, e.g., literature $[10,15]$.

Thus, the above comparison shows that DMs can make decision by choosing different values of the parameters $\beta, \gamma, \eta$ with his/her attitude, which is superior to the traditional operators, and that the developed method in this paper is more objective than the method in literature $[10,15]$.

\section{Conclusions}

In this paper, we develop a new operator called the IGOWUMA operator based on an optimal deviation model, which incorporates the risk attitude of DMs in the aggregation process. We investigate some properties of the IGOWUMA operator and prove that it is commutative, idempotent, monotonicity with respect to the degree of possibility, boundedness with respect to the degree of possibility and monotonicity with respect to the parameter $\lambda$ for the degree of possibility. These properties are suitable for any utility function. Under the HARA utility function, we proposed an IGOWUMA-HARA operator. We discuss its families and found that it included a wide range of aggregation operators. In order to determine the IGOWUMA-HARA operator' weights, a preemptive nonlinear objective programming model is constructed, which can determine a uniform weighting vector to guarantee the uniform standard comparison between the alternatives and measure their fair competition under the condition of valid comparison between various alternatives. Moreover, a new approach for IMAGDM is developed based on the IGOWUMA-HARA operator. This approach can also be applied effectively to different group decision-making problems such as engineering management and financial management, etc. At last, we present an application of the new approach for IMAGDM in an example of an investment selection.

The innovative contributions of the paper are three-fold: (i) two interval information aggregation operators named as the IGOWUMA operator and the IGOWUMA-HARA operator are developed based on utility function. The two operators take into account the risk attitude of DMs in the aggregation process since different DMs may have different risk attitudes. In addition, the latter can degenerate into various operators by adjusting parameters; (ii) A preemptive nonlinear objective programming model is proposed to determine the weights of operator. This model can provide a uniform weighting vector to guarantee the uniform standard comparison between the alternatives and reduce the influence of unfair argument on the aggregated results; (iii) A new approach for IMAGDM is developed based on the IGOWUMA-HARA operator and the preemptive nonlinear objective programming model. The decision process based on utility aggregation operator is more in line with the actual decision behavior of DMs. This approach is effectively applicable to different group decision-making problems such as engineering management and financial management, etc.

From the sensitive analysis and comparative analysis, some solid and practical implications can be obtained. Firstly, using the IGOWUMA -HARA operator to IMAGDM, DMs can choose different values of $\gamma, \beta, \eta$ to express their risk attributes. The absolute risk aversion coefficient $r(x, \gamma, \beta, \eta)$ of DMs decreases with the increase of the value of $\gamma$ or $\eta$, and increases with the increase of the value $\beta$. In addition, the optimal alternative varies with the risk attitudes of the DMs. Secondly, there is a critical value of the absolute risk aversion coefficient on the optimal alternative. No matter how the parameters change, the optimal scheme will not change as long as the risk aversion coefficient is below or above the critical point. In other word, the magnitude relation between the absolute risk aversion coefficient and the critical value will decide which the optimal alternative is. So it is important to find 
the critical value through sensitivity analysis, and then the DM can express their preferences on the alternative by adjusting the parameters.

In further research, it would be very interesting to extend our analysis to the case of more sophisticated situation such as introducing the behavior theory of DMs in the IGOWUMA-HARA operator. Nevertheless, we leave that point to future research, since our methodology cannot be applied to that extended framework, which will result in more sophisticated calculation.

Acknowledgments: This research is supported by the 2017 Special Project of Cultivation and Development of Innovation Base (NO. Z171100002217024) and the Program for New Century Excellent Talents from Ministry of Education of the China (NCET-10-0375), and the Fundamental Research Funds for the Central Universities under No. 12ZX08.

Author Contributions: Jianwei Gao mainly studied the proposed method; Haobo Zhang collected the relative data; Yunna $\mathrm{Wu}$ calculated the result and drafted the paper, and Chuanbo Xu carried out a case study. Finally, Haobo Zhang and Chuanbo formatted the manuscript for submission.

Conflicts of Interest: The authors declare no conflict of interest.

\section{Appendix A}

Proof of Property 3 (Boundedness).

Since $F$ is IGOWUM operator, then:

$$
\begin{gathered}
F\left(\widetilde{x}_{1}, \widetilde{x}_{2}, \cdots, \widetilde{x}_{n}\right)=u^{-1}\left[\left(\sum_{i=1}^{n} w_{i} u^{\lambda}\left(\widetilde{y}_{i}\right) / \sum_{i=1}^{n}\left(w_{i} / u^{\lambda}\left(\widetilde{y}_{i}\right)\right)\right)^{1 / 2 \lambda}\right] \\
=\left[u^{-1}\left\{\left(\sum_{i=1}^{n} w_{i} u^{\lambda}\left(y_{i}^{L}\right) / \sum_{i=1}^{n}\left(w_{i} / u^{\lambda}\left(y_{i}^{L}\right)\right)\right)^{1 / 2 \lambda}\right\}, u^{-1}\left\{\left(\sum_{i=1}^{n} w_{i} u^{\lambda}\left(y_{i}^{U}\right) / \sum_{i=1}^{n}\left(w_{i} / u^{\lambda}\left(y_{i}^{U}\right)\right)\right)^{1 / 2 \lambda}\right\}\right], \\
F\left(\widetilde{t}_{1}, \widetilde{t}_{1}, \cdots, \widetilde{t}_{1}\right)=u^{-1}\left[\left(\sum_{i=1}^{n} w_{i} u^{\lambda}\left(\widetilde{t}_{1}\right)\right)^{1 / \lambda}\right]=\widetilde{t}_{1}=\left[t_{1}^{L}, t_{1}^{U}\right], F\left(\widetilde{t}_{2}, \widetilde{t}_{2}, \cdots, \widetilde{t}_{2}\right)=u^{-1}\left[\left(\sum_{i=1}^{n} w_{i} u^{\lambda}\left(\widetilde{t}_{2}\right)\right)^{1 / \lambda}\right]=\widetilde{t}_{2}=\left[t_{2}^{L}, t_{2}^{U}\right] .
\end{gathered}
$$

For simplicity, let:

$$
\alpha^{L}=u^{-1}\left\{\left(\sum_{i=1}^{n} w_{i} u^{\lambda}\left(y_{i}^{L}\right) / \sum_{i=1}^{n}\left(w_{i} / u^{\lambda}\left(y_{i}^{L}\right)\right)\right)^{1 / 2 \lambda}\right\} \text { and } \alpha^{U}=u^{-1}\left\{\left(\sum_{i=1}^{n} w_{i} u^{\lambda}\left(y_{i}^{U}\right) / \sum_{i=1}^{n}\left(w_{i} / u^{\lambda}\left(y_{i}^{U}\right)\right)\right)^{1 / 2 \lambda}\right\},
$$

We then have:

$$
F\left(\widetilde{x}_{1}, \widetilde{x}_{2}, \cdots, \widetilde{x}_{n}\right)=\left[\alpha^{L}, \alpha^{U}\right]=\widetilde{\alpha} .
$$

In the following, we will complete the proof with two steps.

Step 1. We first proof $\widetilde{t}_{1} \leq F\left(\widetilde{x}_{1}, \widetilde{x}_{2}, \cdots \widetilde{x}_{n}\right)$.

From the above results, we derive $\alpha^{L} \geq t_{1}^{L}$ and $\alpha^{U} \geq t_{1}^{U}$. Then, we can conclude that

(i) If $t_{1}^{L} \leq \alpha^{L} \leq t_{1}^{U} \leq \alpha^{U}$, then from formula (1), we have:

$$
\begin{gathered}
p\left(\widetilde{\alpha} \geq \widetilde{t}_{1}\right)=\max \left\{1-\max \left(\left[t_{1}^{U}-\alpha^{L}\right] /\left[\left(\alpha^{U}-\alpha^{L}\right)+\left(t_{1}^{U}+t_{1}^{L}\right)\right], 0\right), 0\right\} \\
=1-\left(t_{1}^{U}-\alpha^{L}\right) /\left[\left(\alpha^{U}-\alpha^{L}\right)+\left(t_{1}^{U}-t_{1}^{L}\right)\right]=\left(\alpha^{U}-t_{1}^{L}\right) /\left[\left(\alpha^{U}-\alpha^{L}\right)+\left(t_{1}^{U}-t_{1}^{L}\right)\right]
\end{gathered}
$$

and:

$$
p\left(\widetilde{t}_{1} \geq \widetilde{\alpha}\right)=1-p\left(\widetilde{\alpha} \geq \widetilde{t}_{1}\right)=\left(t_{1}^{U}-\alpha^{L}\right) /\left[\left(\alpha^{U}-\alpha^{L}\right)+\left(t_{1}^{U}-t_{1}^{L}\right)\right] .
$$

From $\left(\alpha^{U}-t_{1}^{L}\right) \geq\left(t_{1}^{U}-\alpha^{L}\right)$, we find that $p\left(\widetilde{\alpha} \geq \widetilde{t}_{1}\right) \geq p\left(\widetilde{t}_{1} \geq \widetilde{\alpha}\right)$, therefore, $\widetilde{t}_{1} \leq F\left(\widetilde{\alpha}_{1}, \widetilde{\alpha}_{2}, \cdots \widetilde{\alpha}_{n}\right)$. (ii) If $t_{1}^{L} \leq t_{1}^{U}<\alpha^{L} \leq \alpha^{U}$, then from formula (1), we have:

$$
p\left(\widetilde{\alpha} \geq \widetilde{t}_{1}\right)=\max \left\{1-\max \left(\left[t_{1}^{U}-\alpha^{L}\right] /\left[\left(\alpha^{U}-\alpha^{L}\right)+\left(t_{1}^{U}-t_{1}^{L}\right)\right], 0\right), 0\right\}=1
$$

and:

$$
p\left(\widetilde{t}_{1} \geq \widetilde{\alpha}\right)=1-p\left(\widetilde{\alpha} \geq \widetilde{t}_{1}\right)=0
$$


Note that $p\left(\widetilde{\alpha} \geq \widetilde{t}_{1}\right) \geq p\left(\widetilde{t}_{1} \geq \widetilde{\alpha}\right)$, we then have $\widetilde{t}_{1} \leq F\left(\widetilde{\alpha}_{1}, \widetilde{\alpha}_{2}, \cdots, \widetilde{\alpha}_{n}\right)$.

Step 2. Similar to the proof of Step 1 , we can conclude that $\widetilde{t}_{2} \geq F\left(\widetilde{\alpha}_{1}, \widetilde{\alpha}_{2}, \cdots, \widetilde{\alpha}_{n}\right)$.

The property is proved.

Proof of Property 4 (Monotonicity with respect to the degree of possibility).

Let:

$$
F\left(\widetilde{x}_{1}, \widetilde{x}_{2}, \cdots, \widetilde{x}_{n}\right)=u^{-1}\left[\left(\sum_{i=1}^{n} w_{i}\left(u\left(\widetilde{y}_{i}\right)\right)^{\lambda} / \sum_{i=1}^{n} w_{i} /\left(u\left(\widetilde{y}_{i}\right)\right)^{\lambda}\right)^{1 / 2 \lambda}\right],
$$

and:

$$
F\left(\widetilde{z}_{1}, \widetilde{z}_{2}, \cdots, \widetilde{z}_{n}\right)=u^{-1}\left[\left(\sum_{i=1}^{n} w_{i}\left(u\left(\widetilde{t}_{i}\right)\right)^{\lambda} / \sum_{i=1}^{n} w_{i} /\left(u\left(\widetilde{t}_{i}\right)\right)^{\lambda}\right)^{1 / 2 \lambda}\right] .
$$

If $p\left(\widetilde{x}_{i} \geq \widetilde{z}_{i}\right)=1$, then we have $p\left(\widetilde{y}_{i} \geq \widetilde{t}_{i}\right)=1$ and $\widetilde{y}_{i}^{L} \geq \widetilde{t}_{i}^{U}$. Since $u(x)$ is a monotonically increasing function, we can obtain $u\left(\widetilde{y}_{i}^{L}\right) \geq u\left(\widetilde{t}_{i}^{U}\right)$. In the following, we will complete the proof in two cases.

Case 1. If $\lambda>0$, then we have $\left(\sum_{i=1}^{n} w_{i} u^{\lambda}\left(\widetilde{y}_{i}^{L}\right) / \sum_{i=1}^{n} w_{i} / u^{\lambda}\left(\widetilde{y}_{i}^{L}\right)\right)^{1 / 2 \lambda} \geq\left(\sum_{i=1}^{n} w_{i} u^{\lambda}\left(\widetilde{t}_{i}^{U}\right) / \sum_{i=1}^{n} w_{i} / u^{\lambda}\left(\widetilde{t}_{i}^{U}\right)\right)^{1 / 2 \lambda}$. Since $u^{-1}(x)$ is a monotonically increasing function, we get:

$$
u^{-1}\left(\left(\sum_{i=1}^{n} w_{i} u^{\lambda}\left(\widetilde{y}_{i}^{L}\right) / \sum_{i=1}^{n} w_{i} / u^{\lambda}\left(\widetilde{y}_{i}^{L}\right)\right)^{1 / 2 \lambda}\right) \geq u^{-1}\left(\left(\sum_{i=1}^{n} w_{i} u^{\lambda}\left(\widetilde{t}_{i}^{U}\right) / \sum_{i=1}^{n} w_{i} / u^{\lambda}\left(\widetilde{t}_{i}^{U}\right)\right)^{1 / 2 \lambda}\right) .
$$

Therefore,

$$
p\left(F\left(\widetilde{x}_{1}, \widetilde{x}_{2}, \cdots, \widetilde{x}_{n}\right) \geq F\left(\widetilde{z}_{1}, \widetilde{z}_{2}, \cdots, \widetilde{z}_{n}\right)\right)=1
$$

Case 2. If $\lambda<0$, then we have $\sum_{i=1}^{n} w_{i} u^{\lambda}\left(\widetilde{y}_{i}^{L}\right) \leq \sum_{i=1}^{n} w_{i} u^{\lambda}\left(\widetilde{z}_{i}^{U}\right)$ and $\sum_{i=1}^{n} w_{i} / u^{\lambda}\left(\widetilde{y}_{i}^{L}\right) \geq \sum_{i=1}^{n} w_{i} / u^{\lambda}\left(\widetilde{z}_{i}^{U}\right)$. Thus,

$$
\sum_{i=1}^{n} w_{i} u^{\lambda}\left(\widetilde{y}_{i}^{L}\right) / \sum_{i=1}^{n} w_{i} / u^{\lambda}\left(\widetilde{y}_{i}^{L}\right) \leq \sum_{i=1}^{n} w_{i} u^{\lambda}\left(\widetilde{z}_{i}^{U}\right) / \sum_{i=1}^{n} w_{i} / u^{\lambda}\left(\widetilde{z}_{i}^{U}\right)
$$

Taking the power of $1 / 2 \lambda$ at both ends of the inequality, we can get:

$$
\left(\sum_{i=1}^{n} w_{i} u^{\lambda}\left(\widetilde{y}_{i}^{L}\right) / \sum_{i=1}^{n} w_{i} / u^{\lambda}\left(\widetilde{y}_{i}^{L}\right)\right)^{1 / 2 \lambda} \geq\left(\sum_{i=1}^{n} w_{i} u^{\lambda}\left(\widetilde{z}_{i}^{U}\right) / \sum_{i=1}^{n} w_{i} / u^{\lambda}\left(\widetilde{z}_{i}^{U}\right)\right)^{1 / 2 \lambda} .
$$

Since $u^{-1}(x)$ is a monotonically increasing function, we obtain:

$$
u^{-1}\left(\left(\sum_{i=1}^{n} w_{i} u^{\lambda}\left(\widetilde{y}_{i}^{L}\right) / \sum_{i=1}^{n} w_{i} / u^{\lambda}\left(\widetilde{y}_{i}^{L}\right)\right)^{1 / 2 \lambda}\right) \geq u^{-1}\left(\left(\sum_{i=1}^{n} w_{i} u^{\lambda}\left(\widetilde{z}_{i}^{U}\right) / \sum_{i=1}^{n} w_{i} / u^{\lambda}\left(\widetilde{z}_{i}^{U}\right)\right)^{1 / 2 \lambda}\right) .
$$

Therefore,

$$
p\left(F\left(\widetilde{x}_{1}, \widetilde{x}_{2}, \cdots, \widetilde{x}_{n}\right) \geq F\left(\widetilde{z}_{1}, \widetilde{z}_{2}, \cdots, \widetilde{z}_{n}\right)\right)=1 .
$$

The property is proved. 
Proof of UGOWMA operator: If $\beta=1-\gamma, \eta \rightarrow 0, \gamma \rightarrow 1$ and $\lambda \in(-\infty, 0) \cup(0,+\infty)$, we get that:

$$
\begin{gathered}
\lim _{\gamma \rightarrow 1}\left(\lim _{\eta \rightarrow 0} F\left(\widetilde{x}_{1}, \widetilde{x}_{2}, \cdots, \widetilde{x}_{n}\right)\right)=\lim _{\gamma \rightarrow 1}\left[\lim _{\eta \rightarrow 0}\left(\left(\sum_{i=1}^{n} w_{i}\left(\widetilde{y}_{i}+\eta\right)^{\lambda \gamma}\right) /\left(\sum_{i=1}^{n} w_{i} /\left(\widetilde{y}_{i}+\eta\right)^{\lambda \gamma}\right)\right)^{1 / 2 \lambda \gamma}-\eta\right] \\
=\lim _{\gamma \rightarrow 1}\left(\sum_{i=1}^{n} w_{i}\left(\widetilde{y}_{i}\right)^{\lambda \gamma} /\left(\sum_{i=1}^{n} w_{i} /\left(\widetilde{y}_{i}\right)^{\lambda \gamma}\right)\right)^{1 / 2 \lambda \gamma}=\left(\sum_{i=1}^{n} w_{i} \widetilde{y}_{i}^{\lambda} /\left(\sum_{i=1}^{n} w_{i} / \widetilde{y}_{i}^{\lambda}\right)\right)^{1 / 2 \lambda} .
\end{gathered}
$$

The theorem is proved.

Proof of UHUG operator: Let $F$ be the UHUM operator, we have:

$$
\begin{gathered}
F(\lambda)=\frac{1-\gamma}{\beta}\left[\left(\sum_{i=1}^{n} w_{i}\left(\frac{\beta}{1-\gamma} \widetilde{x}_{i}+\eta\right)^{\lambda \gamma} / \sum_{i=1}^{n}\left(w_{i} /\left(\frac{\beta}{1-\gamma} \widetilde{x}_{i}+\eta\right)^{\lambda \gamma}\right)\right)^{1 / 2 \lambda \gamma}-\eta\right] \\
=\left[\frac{1-\gamma}{\beta}\left(\left(\sum_{i=1}^{n} w_{i}\left(\frac{\beta}{1-\gamma} \widetilde{y}_{i}^{L}+\eta\right)^{\lambda \gamma} / \sum_{i=1}^{n}\left(w_{i} /\left(\frac{\beta}{1-\gamma} \widetilde{y}_{i}^{L}+\eta\right)\right)^{\lambda \gamma}-\eta\right), \frac{1-\gamma}{\beta}\left(\left(\sum_{i=1}^{n} w_{i}\left(\frac{\beta}{1-\gamma} \widetilde{y}_{i}^{U}+\eta\right)^{\lambda \gamma} / \sum_{i=1}^{n}\left(w_{i} /\left(\frac{\beta}{1-\gamma} \widetilde{y}_{i}^{U}+\eta\right)^{\lambda \gamma}\right)\right)^{1 / 2 \lambda \gamma}-\eta\right)\right] .\right.
\end{gathered}
$$

In the following, we will complete the proof in two cases.

Case 1. If $\lambda \rightarrow 0^{+}$, then:

$$
\begin{aligned}
& \lim _{\lambda \rightarrow 0^{+}} F(\lambda)=\left[\frac{1-\gamma}{\beta}\left(\left(\sum_{i=1}^{n} w_{i}\left(\frac{\beta}{1-\gamma} \widetilde{y}_{i}^{L}+\eta\right)^{\lambda \gamma} / \sum_{i=1}^{n}\left(w_{i} /\left(\frac{\beta}{1-\gamma} \widetilde{y}_{i}^{L}+\eta\right)^{\lambda \gamma}\right)\right)^{1 / 2 \lambda \gamma}-\eta\right), \frac{1-\gamma}{\beta}\left(\left(\sum_{i=1}^{n} w_{i}\left(\frac{\beta}{1-\gamma} \widetilde{y}_{i}^{U}+\eta\right)^{\lambda \gamma} / \sum_{i=1}^{n}\left(w_{i} /\left(\frac{\beta}{1-\gamma} \widetilde{y}_{i}^{U}+\eta\right)^{\lambda \gamma}\right)\right)^{1 / 2 \lambda \gamma}-\eta\right)\right] \\
& =\left[\lim _{\lambda \rightarrow 0^{+}} \frac{1-\gamma}{\beta}\left(\left(\sum_{i=1}^{n} w_{i}\left(\frac{\beta}{1-\gamma} \widetilde{y}_{i}^{L}+\eta\right)^{\lambda \gamma} / \sum_{i=1}^{n}\left(w_{i} /\left(\frac{\beta}{1-\gamma} \widetilde{y}_{i}^{L}+\eta\right)^{\lambda \gamma}\right)\right)^{1 / 2 \lambda \gamma}-\eta\right), \lim _{\lambda \rightarrow 0^{+}} \frac{1-\gamma}{\beta}\left(\left(\sum_{i=1}^{n} w_{i}\left(\frac{\beta}{1-\gamma} \widetilde{y}_{i}^{U}+\eta\right)^{\lambda \gamma} / \sum_{i=1}^{n}\left(w_{i} /\left(\frac{\beta}{1-\gamma} \widetilde{y}_{i}^{U}+\eta\right)^{\lambda \gamma}\right)\right)^{1 / 2 \lambda \gamma}-\eta\right)\right] .
\end{aligned}
$$

By the L'Hôpital's rule, we have that:

$$
\begin{gathered}
\lim _{\lambda \rightarrow 0^{+}}\left(\sum_{i=1}^{n} w_{i}\left(\frac{\beta}{1-\gamma} \widetilde{y}_{i}^{L}+\eta\right)^{\lambda \gamma} / \sum_{i=1}^{n}\left(w_{i} /\left(\frac{\beta}{1-\gamma} \widetilde{y}_{i}^{L}+\eta\right)^{\lambda \gamma}\right)\right)^{1 / 2 \lambda \gamma} \\
=\lim _{\lambda \rightarrow 0^{+}} \exp \left(\frac{1}{2 \lambda \gamma} \log \sum_{i=1}^{n} w_{i}\left(\frac{\beta}{1-\gamma} \widetilde{y}_{i}^{L}+\eta\right)^{\lambda \gamma} / \sum_{i=1}^{n}\left(w_{i} /\left(\frac{\beta}{1-\gamma} \widetilde{y}_{i}^{L}+\eta\right)^{\lambda \gamma}\right)\right) \\
=\exp \left(\sum_{i=1}^{n} w_{i} \log \left(\frac{\beta}{1-\gamma} \widetilde{y}_{i}^{L}+\eta\right)\right)=\prod_{i=1}^{n}\left(\frac{\beta}{1-\gamma} \widetilde{y}_{i}^{L}+\eta\right)^{w_{i}} .
\end{gathered}
$$

Similarly, we can get:

$$
\lim _{\lambda \rightarrow 0^{+}}\left(\sum_{i=1}^{n} w_{i}\left(\frac{\beta}{1-\gamma} \widetilde{y}_{i}^{u}+\eta\right)^{\lambda \gamma} / \sum_{i=1}^{n}\left(w_{i} /\left(\frac{\beta}{1-\gamma} \widetilde{y}_{i}^{u}+\eta\right)^{\lambda \gamma}\right)\right)^{1 / 2 \lambda \gamma}=\prod_{i=1}^{n}\left(\frac{\beta}{1-\gamma} \widetilde{y}_{i}^{u}+\eta\right)^{w_{i}} .
$$

Thus,

$$
\lim _{\lambda \rightarrow 0^{+}} F(\lambda)=\left[\frac{1-\gamma}{\beta}\left(\prod_{i=1}^{n}\left(\frac{\beta}{1-\gamma} \widetilde{y}_{i}^{L}+\eta\right)^{w_{i}}-\eta\right), \frac{1-\gamma}{\beta}\left(\prod_{i=1}^{n}\left(\frac{\beta}{1-\gamma} \widetilde{y}_{i}^{U}+\eta\right)^{w_{i}}-\eta\right)\right] .
$$

That is,

$$
\lim _{\lambda \rightarrow 0^{+}} F\left(\widetilde{x}_{1}, \widetilde{x}_{2}, \cdots, \widetilde{x}_{n}\right)=\frac{1-\gamma}{\beta}\left(\prod_{i=1}^{n}\left(\frac{\beta}{1-\gamma} \widetilde{y}_{i}+\eta\right)^{w_{i}}-\eta\right) .
$$

Case 2. If $\lambda \rightarrow 0^{-1}$, then similar to the above proof.

The theorem is proved.

Proof of ULGA operator: Let $f$ be the UHUMA operator, we have:

$$
\begin{gathered}
f(\gamma)=\frac{1-\gamma}{\beta}\left[\left(\sum_{i=1}^{n} w_{i}\left(\frac{\beta}{1-\gamma} \widetilde{y}_{i}+\eta\right)^{\gamma} / \sum_{i=1}^{n}\left(w_{i} /\left(\frac{\beta}{1-\gamma} \widetilde{y}_{i}+\eta\right)^{\gamma}\right)\right)^{1 / 2 \gamma}-n\right] \\
=\left[\frac{1-\gamma}{\beta}\left(\left(\sum_{i=1}^{n} w_{i}\left(\frac{\beta}{1-\gamma} \widetilde{\gamma}_{i}^{L}+\eta\right)^{\gamma} / \sum_{i=1}^{n}\left(w_{i} /\left(\frac{\beta}{1-\gamma} \tilde{y}_{i}^{L}+\eta\right)^{\gamma}\right)\right)^{1 / 2 \gamma}-n\right), \frac{1-\gamma}{\beta}\left(\left(\sum_{i=1}^{n} w_{i}\left(\frac{\beta}{1-\gamma} \widetilde{y}_{i}^{U}+\eta\right)^{\gamma} / \sum_{i=1}^{n}\left(w_{i} /\left(\frac{\beta}{1-\gamma} \widetilde{y}_{i}^{U}+\eta\right)^{\gamma}\right)\right)^{1 / 2 \gamma}-n\right)\right] .
\end{gathered}
$$


In the following, we will complete the proof in two cases.

Case 1. If $\gamma \rightarrow 0^{+}$, then:

$$
\begin{gathered}
\lim _{\gamma \rightarrow 0^{+}} f(\lambda)=\lim _{\gamma \rightarrow 0^{+}}\left[\frac{1-\gamma}{\beta}\left(\left(\sum_{i=1}^{n} w_{i}\left(\frac{\beta}{1-\gamma} \tilde{y}_{i}^{L}+\eta\right)^{\gamma} / \sum_{i=1}^{n}\left(w_{i} /\left(\frac{\beta}{1-\gamma} \tilde{y}_{i}^{L}+\eta\right)^{\gamma}\right)\right)^{1 / 2 \gamma}-n\right), \frac{1-\gamma}{\beta}\left(\left(\sum_{i=1}^{n} w_{i}\left(\frac{\beta}{1-\gamma} \widetilde{y}_{i}^{U}+\eta\right)^{\gamma} / \sum_{i=1}^{n}\left(w_{i} /\left(\frac{\beta}{1-\gamma} \widetilde{y}_{i}^{U}+\eta\right)^{\gamma}\right)\right)^{1 / 2 \gamma}-n\right)\right] \\
=\left[\lim _{\gamma \rightarrow 0^{+}} \frac{1-\gamma}{\beta}\left(\left(\sum_{i=1}^{n} w_{i}\left(\frac{\beta}{1-\gamma} \tilde{y}_{i}^{L}+\eta\right)^{\gamma} / \sum_{i=1}^{n}\left(w_{i} /\left(\frac{\beta}{1-\gamma} \widetilde{y}_{i}^{L}+\eta\right)^{\gamma}\right)\right)^{1 / 2 \gamma}-n\right), \lim _{\gamma \rightarrow 0^{+}} \frac{1-\gamma}{\beta}\left(\left(\sum_{i=1}^{n} w_{i}\left(\frac{\beta}{1-\gamma} \widetilde{y}_{i}^{U}+\eta\right)^{\gamma} / \sum_{i=1}^{n}\left(w_{i} /\left(\frac{\beta}{1-\gamma} \widetilde{\gamma}_{i}^{U}+\eta\right)^{\gamma}\right)\right)^{1 / 2 \gamma}-n\right)\right] .
\end{gathered}
$$

By the L'Hôpital's rule, we have that:

$$
\begin{gathered}
\lim _{\gamma \rightarrow 0^{+}}\left(\sum_{i=1}^{n} w_{i}\left(\frac{\beta}{1-\gamma} \widetilde{y}_{i}^{L}+\eta\right)^{\gamma} / \sum_{i=1}^{n}\left(w_{i} /\left(\frac{\beta}{1-\gamma} \widetilde{y}_{i}^{L}+\eta\right)^{\gamma}\right)\right)^{1 / 2 \gamma} \\
=\lim _{\gamma \rightarrow 0^{+}} \exp \left(\frac{1}{2 \gamma} \log \sum_{i=1}^{n} w_{i}\left(\frac{\beta}{1-\gamma} \widetilde{y}_{i}^{L}+\eta\right)^{\gamma} / \sum_{i=1}^{n}\left(w_{i} /\left(\frac{\beta}{1-\gamma} \widetilde{y}_{i}^{L}+\eta\right)^{\gamma}\right)\right) \\
=\exp \left(\sum_{i=1}^{n} w_{i} \log \left(\beta \widetilde{y}_{i}^{L}+\eta\right)\right)=\prod_{i=1}^{n}\left(\beta \widetilde{y}_{i}^{L}+\eta\right)^{w_{i}} .
\end{gathered}
$$

Similarly, we can get:

$$
\lim _{\gamma \rightarrow 0^{+}}\left(\sum_{i=1}^{n} w_{i}\left(\frac{\beta}{1-\gamma} \widetilde{y}_{i}^{U}+\eta\right)^{\gamma} / \sum_{i=1}^{n}\left(w_{i} /\left(\frac{\beta}{1-\gamma} \widetilde{y}_{i}^{U}+\eta\right)^{\gamma}\right)\right)^{1 / 2 \gamma}=\prod_{i=1}^{n}\left(\beta \widetilde{y}_{i}^{U}+\eta\right)^{w_{i}} .
$$

Thus,

$$
\lim _{\gamma \rightarrow 0^{+}} f(\lambda)=\left[\frac{1}{\beta}\left(\prod_{i=1}^{n}\left(\beta \widetilde{y}_{i}^{L}+\eta\right)^{w_{i}}-\eta\right), \frac{1}{\beta}\left(\prod_{i=1}^{n}\left(\beta \widetilde{y}_{i}^{U}+\eta\right)^{w_{i}}-\eta\right)\right] .
$$

That is,

$$
\lim _{\gamma \rightarrow 0^{+}} f\left(\widetilde{x}_{1}, \widetilde{x}_{2}, \cdots, \widetilde{x}_{n}\right)=\frac{1}{\beta}\left(\prod_{i=1}^{n}\left(\beta \widetilde{y}_{i}+\eta\right)^{w_{i}}-\eta\right)
$$

Case 2. If $\lambda \rightarrow 0^{-1}$, then similar to the above proof.

The theorem is proved.

\section{Appendix B}

Table A1. Decision matrix $\widetilde{B}^{(1)}-d_{1}$.

\begin{tabular}{cccc}
\hline$x_{\boldsymbol{i}}$ & $\boldsymbol{c}_{\boldsymbol{1}}$ & $\boldsymbol{c}_{\mathbf{2}}$ & $\boldsymbol{c}_{\mathbf{3}}$ \\
\hline$x_{1}$ & {$[80,90]$} & {$[95,100]$} & {$[70,80]$} \\
$x_{2}$ & {$[75,85]$} & {$[85,95]$} & {$[40,50]$} \\
$x_{3}$ & {$[80,100]$} & {$[75,80]$} & {$[85,90]$} \\
$x_{4}$ & {$[60,80]$} & {$[85,90]$} & {$[75,80]$} \\
$x_{5}$ & {$[70,75]$} & {$[95,100]$} & {$[80,90]$} \\
$x_{6}$ & {$[90,95]$} & {$[60,70]$} & {$[90,100]$} \\
\hline
\end{tabular}

Table A2. Decision matrix $\widetilde{B}^{(2)}-d_{2}$.

\begin{tabular}{cccc}
\hline$x_{\boldsymbol{i}}$ & $\boldsymbol{c}_{\mathbf{1}}$ & $\boldsymbol{c}_{\mathbf{2}}$ & $\boldsymbol{c}_{\mathbf{3}}$ \\
\hline$x_{1}$ & {$[90,100]$} & {$[80,90]$} & {$[40,50]$} \\
$x_{2}$ & {$[90,95]$} & {$[75,80]$} & {$[85,95]$} \\
$x_{3}$ & {$[85,95]$} & {$[70,85]$} & {$[90,95]$} \\
$x_{4}$ & {$[90,95]$} & {$[75,80]$} & {$[90,95]$} \\
$x_{5}$ & {$[80,85]$} & {$[90,95]$} & {$[85,90]$} \\
$x_{6}$ & {$[80,95]$} & {$[75,80]$} & {$[90,95]$} \\
\hline
\end{tabular}


Table A3. Decision matrix $\widetilde{B}^{(3)}-d_{3}$.

\begin{tabular}{cccc}
\hline$x_{\boldsymbol{i}}$ & $\boldsymbol{c}_{\mathbf{1}}$ & $\boldsymbol{c}_{\mathbf{2}}$ & $\boldsymbol{c}_{\mathbf{3}}$ \\
\hline$x_{1}$ & {$[85,95]$} & {$[90,95]$} & {$[85,90]$} \\
$x_{2}$ & {$[80,90]$} & {$[85,90]$} & {$[70,75]$} \\
$x_{3}$ & {$[85,95]$} & {$[85,90]$} & {$[80,85]$} \\
$x_{4}$ & {$[90,100]$} & {$[80,95]$} & {$[85,90]$} \\
$x_{5}$ & {$[85,95]$} & {$[90,100]$} & {$[80,85]$} \\
$x_{6}$ & {$[90,100]$} & {$[55,60]$} & {$[85,95]$} \\
\hline
\end{tabular}

Table A4. Decision matrix $\widetilde{B}^{(4)}-d_{4}$.

\begin{tabular}{cccc}
\hline$x_{\boldsymbol{i}}$ & $\boldsymbol{c}_{\mathbf{1}}$ & $\boldsymbol{c}_{\mathbf{2}}$ & $\boldsymbol{c}_{\mathbf{3}}$ \\
\hline$x_{1}$ & {$[80,85]$} & {$[90,95]$} & {$[80,90]$} \\
$x_{2}$ & {$[75,90]$} & {$[95,100]$} & {$[75,85]$} \\
$x_{3}$ & {$[85,95]$} & {$[90,95]$} & {$[85,90]$} \\
$x_{4}$ & {$[85,90]$} & {$[80,85]$} & {$[90,100]$} \\
$x_{5}$ & {$[90,95]$} & {$[85,90]$} & {$[75,80]$} \\
$x_{6}$ & {$[85,90]$} & {$[90,95]$} & {$[80,90]$} \\
\hline
\end{tabular}

Table A5. The best alternative under the changing of parameters $\gamma, \eta, \beta$.

\begin{tabular}{|c|c|c|c|c|c|c|c|c|c|c|c|}
\hline \multirow{2}{*}{ No. } & \multirow{2}{*}{$\gamma$} & \multirow{2}{*}{$\eta$} & \multirow{2}{*}{$\beta$} & \multirow{2}{*}{$r(x)$} & \multicolumn{6}{|c|}{$p_{i}(i=1,2, \cdots 6)$} & \multirow[t]{2}{*}{ Ordering } \\
\hline & & & & & $p_{1}$ & $p_{2}$ & $p_{3}$ & $p_{4}$ & $p_{5}$ & $p_{6}$ & \\
\hline 1 & 0.8 & 1 & 3 & 0.353 & 0.17376 & 0.14469 & 0.18543 & 0.16269 & 0.18560 & 0.14784 & $x_{5} \succ x_{3} \succ x_{1} \succ x_{4} \succ x_{6} \succ x_{2}$ \\
\hline 2 & 0.4 & 1 & 3 & 0.857 & 0.17384 & 0.14479 & 0.18579 & 0.16218 & 0.18593 & 0.14747 & $x_{5} \succ x_{3} \succ x_{1} \succ x_{4} \succ x_{6} \succ x_{2}$ \\
\hline 3 & -0.5 & 1 & 3 & 1.500 & 0.17347 & 0.14456 & 0.18640 & 0.16192 & 0.18636 & 0.14728 & $x_{3} \succ x_{5} \succ x_{1} \succ x_{4} \succ x_{6} \succ x_{2}$ \\
\hline 4 & -1.4 & 1 & 3 & 1.846 & 0.17203 & 0.14353 & 0.18726 & 0.16251 & 0.18695 & 0.14772 & $x_{3} \succ x_{5} \succ x_{1} \succ x_{4} \succ x_{6} \succ x_{2}$ \\
\hline 5 & -2 & 1 & 3 & 2.000 & 0.17047 & 0.14234 & 0.18801 & 0.16315 & 0.18782 & 0.14820 & $x_{3} \succ x_{5} \succ x_{1} \succ x_{4} \succ x_{6} \succ x_{2}$ \\
\hline 6 & 0.1 & 2.7 & 3 & 0.687 & 0.17391 & 0.14485 & 0.18590 & 0.16198 & 0.18601 & 0.14735 & $x_{5} \succ x_{3} \succ x_{1} \succ x_{4} \succ x_{6} \succ x_{2}$ \\
\hline 7 & 0.1 & 1.7 & 3 & 0.891 & 0.17382 & 0.14480 & 0.18600 & 0.16195 & 0.18613 & 0.14730 & $x_{5} \succ x_{3} \succ x_{1} \succ x_{4} \succ x_{6} \succ x_{2}$ \\
\hline 8 & 0.1 & 1.2 & 3 & 1.047 & 0.17378 & 0.14476 & 0.18608 & 0.16193 & 0.18616 & 0.14728 & $x_{5} \succ x_{3} \succ x_{1} \succ x_{4} \succ x_{6} \succ x_{2}$ \\
\hline 9 & 0.1 & 0.8 & 3 & 1.216 & 0.17371 & 0.14472 & 0.18623 & 0.16188 & 0.18621 & 0.14724 & $x_{3} \succ x_{5} \succ x_{1} \succ x_{4} \succ x_{6} \succ x_{2}$ \\
\hline 10 & 0.1 & 0.4 & 3 & 1.452 & 0.17367 & 0.14470 & 0.18630 & 0.16187 & 0.18624 & 0.14722 & $x_{3} \succ x_{5} \succ x_{1} \succ x_{4} \succ x_{6} \succ x_{2}$ \\
\hline 11 & 0.1 & 1 & 0.5 & 0.391 & 0.17406 & 0.14496 & 0.18574 & 0.16199 & 0.18584 & 0.14741 & $x_{5} \succ x_{3} \succ x_{1} \succ x_{4} \succ x_{6} \succ x_{2}$ \\
\hline 12 & 0.1 & 1 & 1.9 & 0.924 & 0.17385 & 0.14481 & 0.18593 & 0.16197 & 0.18611 & 0.14732 & $x_{5} \succ x_{3} \succ x_{1} \succ x_{4} \succ x_{6} \succ x_{2}$ \\
\hline 13 & 0.1 & 1 & 2.5 & 1.047 & 0.17382 & 0.14479 & 0.18597 & 0.16196 & 0.18615 & 0.14731 & $x_{5} \succ x_{3} \succ x_{1} \succ x_{4} \succ x_{6} \succ x_{2}$ \\
\hline 14 & 0.1 & 1 & 3.6 & 1.200 & 0.17367 & 0.14470 & 0.186283 & 0.16185 & 0.186278 & 0.14721 & $x_{3} \succ x_{5} \succ x_{1} \succ x_{4} \succ x_{6} \succ x_{2}$ \\
\hline 15 & 0.1 & 1 & 5 & 1.324 & 0.17359 & 0.14465 & 0.18645 & 0.16179 & 0.18637 & 0.14716 & $x_{3} \succ x_{5} \succ x_{1} \succ x_{4} \succ x_{6} \succ x_{2}$ \\
\hline 16 & 0.9 & 1 & 0.2 & 0.100 & 0.17294 & 0.14420 & 0.18628 & 0.16210 & 0.18706 & 0.14741 & $x_{5} \succ x_{3} \succ x_{1} \succ x_{4} \succ x_{6} \succ x_{2}$ \\
\hline 17 & 0.3 & 1 & 1.9 & 0.806 & 0.17356 & 0.14463 & 0.18600 & 0.16183 & 0.18676 & 0.14721 & $x_{5} \succ x_{3} \succ x_{1} \succ x_{4} \succ x_{6} \succ x_{2}$ \\
\hline 18 & -1.9 & 1 & 3.3 & 2.103 & 0.17098 & 0.14269 & 0.18754 & 0.16325 & 0.18726 & 0.14828 & $x_{3} \succ x_{5} \succ x_{1} \succ x_{4} \succ x_{6} \succ x_{2}$ \\
\hline 19 & -0.8 & 1 & 4.1 & 1.917 & 0.17329 & 0.14442 & 0.18646 & 0.16223 & 0.18610 & 0.14750 & $x_{3} \succ x_{5} \succ x_{1} \succ x_{4} \succ x_{6} \succ x_{2}$ \\
\hline 20 & -0.2 & 1 & 4.7 & 1.589 & 0.17381 & 0.14478 & 0.18623 & 0.16200 & 0.18585 & 0.14733 & $x_{3} \succ x_{5} \succ x_{1} \succ x_{4} \succ x_{6} \succ x_{2}$ \\
\hline 21 & 0.8 & 3 & 3 & 0.286 & 0.17313 & 0.14432 & 0.18632 & 0.16212 & 0.18670 & 0.14741 & $x_{5} \succ x_{3} \succ x_{1} \succ x_{4} \succ x_{6} \succ x_{2}$ \\
\hline 22 & 0.4 & 2.4 & 3 & 0.612 & 0.17362 & 0.14466 & 0.18614 & 0.16195 & 0.18634 & 0.14730 & $x_{5} \succ x_{3} \succ x_{1} \succ x_{4} \succ x_{6} \succ x_{2}$ \\
\hline 23 & 0.2 & 1.3 & 3 & 0.945 & 0.17371 & 0.14472 & 0.18610 & 0.16191 & 0.18630 & 0.14726 & $x_{5} \succ x_{3} \succ x_{1} \succ x_{4} \succ x_{6} \succ x_{2}$ \\
\hline 24 & -0.8 & 0.6 & 3 & 2.093 & 0.17323 & 0.14438 & 0.18640 & 0.16218 & 0.18633 & 0.14747 & $x_{3} \succ x_{5} \succ x_{1} \succ x_{4} \succ x_{6} \succ x_{2}$ \\
\hline 25 & -1.7 & 0.3 & 3 & 3.507 & 0.17138 & 0.14301 & 0.18731 & 0.16303 & 0.18718 & 0.14809 & $x_{3} \succ x_{5} \succ x_{1} \succ x_{4} \succ x_{6} \succ x_{2}$ \\
\hline 26 & 0.1 & 0.7 & 0.3 & 0.346 & 0.17378 & 0.14481 & 0.18585 & 0.16172 & 0.18663 & 0.14722 & $x_{5} \succ x_{3} \succ x_{1} \succ x_{4} \succ x_{6} \succ x_{2}$ \\
\hline 27 & 0.1 & 2.5 & 1.3 & 0.403 & 0.17375 & 0.14478 & 0.18588 & 0.16174 & 0.18665 & 0.14721 & $x_{5} \succ x_{3} \succ x_{1} \succ x_{4} \succ x_{6} \succ x_{2}$ \\
\hline 28 & 0.1 & 0.2 & 0.8 & 1.241 & 0.17378 & 0.14476 & 0.18615 & 0.16195 & 0.18670 & 0.14729 & $x_{5} \succ x_{3} \succ x_{1} \succ x_{4} \succ x_{6} \succ x_{2}$ \\
\hline 29 & 0.1 & 1.1 & 2.9 & 1.069 & 0.17378 & 0.14477 & 0.18613 & 0.16193 & 0.18611 & 0.14728 & $x_{3} \succ x_{5} \succ x_{1} \succ x_{4} \succ x_{6} \succ x_{2}$ \\
\hline 30 & 0.1 & 2.3 & 4.9 & 0.976 & 0.17379 & 0.14477 & 0.18614 & 0.16192 & 0.18605 & 0.14729 & $x_{3} \succ x_{5} \succ x_{1} \succ x_{4} \succ x_{6} \succ x_{2}$ \\
\hline 31 & 0.65 & 3.3 & 1.4 & 0.264 & 0.17339 & 0.14451 & 0.18620 & 0.16201 & 0.18653 & 0.14736 & $x_{5} \succ x_{3} \succ x_{1} \succ x_{4} \succ x_{6} \succ x_{2}$ \\
\hline 32 & 0.25 & 1.4 & 0.4 & 0.240 & 0.17389 & 0.14487 & 0.18596 & 0.16180 & 0.18617 & 0.14731 & $x_{5} \succ x_{3} \succ x_{1} \succ x_{4} \succ x_{6} \succ x_{2}$ \\
\hline 33 & -0.4 & 1.6 & 4.8 & 1.448 & 0.17368 & 0.14469 & 0.18621 & 0.16200 & 0.18607 & 0.14734 & $x_{3} \succ x_{5} \succ x_{1} \succ x_{4} \succ x_{6} \succ x_{2}$ \\
\hline 34 & -0.9 & 1.9 & 5 & 1.555 & 0.17312 & 0.14431 & 0.18644 & 0.16223 & 0.18638 & 0.14752 & $x_{3} \succ x_{5} \succ x_{1} \succ x_{4} \succ x_{6} \succ x_{2}$ \\
\hline 35 & -1.5 & 0.5 & 3.4 & 2.881 & 0.17194 & 0.14342 & 0.18710 & 0.16282 & 0.18678 & 0.14794 & $x_{3} \succ x_{5} \succ x_{1} \succ x_{4} \succ x_{6} \succ x_{2}$ \\
\hline
\end{tabular}




\section{References}

1. Qin, J.; Liu, X.; Pedrycz, W. An extended todim multi-criteria group decision making method for green supplier selection in interval type-2 fuzzy environment. Eur. J. Oper. Res. 2017, 258, 626-638. [CrossRef]

2. Ren, Z.; Xu, Z.; Wang, H. Dual hesitant fuzzy vikor method for multi-criteria group decision making based on fuzzy measure and new comparison method. Inf. Sci. 2017, 388, 1-16. [CrossRef]

3. Liu, J.; Chen, H.; Xu, Q.; Zhou, L.; Tao, Z. Generalized ordered modular averaging operator and its application to group decision making. Fuzzy Sets Syst. 2016, 299, 1-25. [CrossRef]

4. Merigó, J.M.; Palacios-Marqués, D.; Zeng, S. Subjective and objective information in linguistic multi-criteria group decision making. Eur. J. Oper. Res. 2016, 248, 522-531. [CrossRef]

5. Liu, Y.; Fan, Z.-P.; Zhang, X. A method for large group decision-making based on evaluation information provided by participators from multiple groups. Inf. Fusion 2016, 29, 132-141. [CrossRef]

6. Gao, J.; Li, M.; Liu, H. Generalized ordered weighted utility averaging-hyperbolic absolute risk aversion operators and their applications to group decision-making. Eur. J Oper. Res. 2015, 243, 258-270. [CrossRef]

7. Gao, J.; Li, M.; Liu, H. Generalized ordered weighted utility proportional averaging-hyperbolic absolute risk aversion operators and their applications to group decision-making. Appl. Math. Comput. 2015, 252, 114-132. [CrossRef]

8. Yager, R.R. On ordered weighted averaging aggregation operators in multicriteria decisionmaking. IEEE Trans. Syst. Man Cybern. 1988, 18, 183-190. [CrossRef]

9. Xu, Z.; Da, Q.-L. The uncertain owa operator. Int. J. Intell. Syst. 2002, 17, 569-575. [CrossRef]

10. $\mathrm{Xu}, \mathrm{Z}$. Dependent uncertain ordered weighted aggregation operators. Inf. Fusion 2008, 9, 310-316. [CrossRef]

11. $\mathrm{Xu}, \mathrm{Z}$; Y Yager, R.R. Power-geometric operators and their use in group decision making. IEEE Trans. Fuzzy Syst. 2010, 18, 94-105.

12. Merigó, J.M.; Casanovas, M. The uncertain induced quasi-arithmetic OWA operator. Int. J. Intell. Syst. 2011, 26, 1-24. [CrossRef]

13. Merigó, J.M.; Casanovas, M. Induced and uncertain heavy OWA operators. Comput. Ind. Eng. 2011, 60, 106-116. [CrossRef]

14. $\mathrm{Xu}, \mathrm{Z}$.; Cai, $\mathrm{X}$. Uncertain power average operators for aggregating interval fuzzy preference relations. Group Decis. Negot. 2012, 21, 381-397. [CrossRef]

15. Zhou, L.-G.; Chen, H.-Y.; Merigó, J.M.; Gil-Lafuente, A.M. Uncertain generalized aggregation operators. Expert Syst. Appl. 2012, 39, 1105-1117. [CrossRef]

16. Zeng, S.; Merigó, J.M.; Su, W. The uncertain probabilistic OWA distance operator and its application in group decision making. Appl. Math. Model. 2013, 37, 6266-6275. [CrossRef]

17. Zhou, S.-M.; Chiclana, F.; John, R.I.; Garibaldi, J.M. Type-1 OWA operators for aggregating uncertain information with uncertain weights induced by type-2 linguistic quantifiers. Fuzzy Sets Syst. 2008, 159, 3281-3296. [CrossRef]

18. Ahn, B.S. The uncertain OWA aggregation with weighting functions having a constant level of orness. Int. J. Intell. Syst. 2006, 21, 469-483. [CrossRef]

19. Xu, Z. A C-OWA operator-based approach to decision making with interval fuzzy preference relation. Int. J. Intell. Syst. 2006, 21, 1289-1298. [CrossRef]

20. Moore, R.E. Interval Analysis; Prentice-Hall: Englewood Cliffs, NJ, USA, 1966; Volume 4.

21. Bustince, H.; Fernández, J.; Kolesárová, A.; Mesiar, R. Generation of linear orders for intervals by means of aggregation functions. Fuzzy Sets Syst. 2013, 220, 69-77. [CrossRef]

22. Grasselli, M. A stability result for the HARA class with stochastic interest rates. Insur. Math. Econ. 2003, 33, 611-627. [CrossRef]

23. Jung, E.J.; Kim, J.H. Optimal investment strategies for the HARA utility under the constant elasticity of variance model. Insur. Math. Econ. 2012, 51, 667-673. [CrossRef]

24. Merton, R.C. Optimum consumption and portfolio rules in a continuous-time model. J. Econ. Theory 1971, 3, 373-413. [CrossRef]

25. Cox, J.C.; Huang, C.-F. Optimal consumption and portfolio policies when asset prices follow a diffusion process. J. Econ. Theory 1989, 49, 33-83. [CrossRef]

26. Calvo, T.; Beliakov, G. Aggregation functions based on penalties. Fuzzy Sets Syst. 2010, 161, 1420-1436. [CrossRef] 
27. Calvo, T.; Mesiar, R.; Yager, R.R. Quantitative weights and aggregation. IEEE Trans. Fuzzy Syst. 2004, 12, 62-69. [CrossRef]

28. Grabisch, M.; Marichal, J.-L.; Mesiar, R.; Pap, E. Aggregation functions: Means. Inf. Sci. 2011, 181, 1-22. [CrossRef]

29. Zhou, L.; Chen, H.; Liu, J. Generalized multiple averaging operators and their applications to group decision making. Group Decis. Negot. 2013, 22, 331-358. [CrossRef]

30. Beliakov, G.; Bustince, H.; Paternain, D. Image reduction using means on discrete product lattices. IEEE Trans. Image Process. 2012, 21, 1070-1083. [CrossRef] [PubMed]

31. Xu, Z. Uncertain Multiple Attribute Decision Making: Methods and Applications; Tsinghua University Press: Beijing, China, 2004.

32. Baird, B.F. Managerial Decisions under Uncertainty: An Introduction to the Analysis of Decision Making; John Wiley \& Sons: Hoboken, NJ, USA, 1989; Volume 4.

(C) 2017 by the authors. Licensee MDPI, Basel, Switzerland. This article is an open access article distributed under the terms and conditions of the Creative Commons Attribution (CC BY) license (http:/ / creativecommons.org/licenses/by/4.0/). 